\title{
MADMatch: Many-to-many Approximate Diagram Matching for design comparison
}

\author{
Segla Kpodjedo, Filippo Ricca, Philippe Galinier, Giuliano Antoniol, and Yann-Gaël Guéhéneuc
}

\begin{abstract}
Matching algorithms play a fundamental role in many important but difficult software engineering activities, especially design evolution analysis and model comparison. We present MADMatch, a fast and scalable Many-to-many Approximate Diagram Matching approach based on an Error-Tolerant Graph matching (ETGM) formulation. Diagrams are represented as graphs, costs are assigned to possible differences between two given graphs, and the goal is to retrieve the cheapest matching. We address the resulting optimisation problem with a tabu search enhanced by the novel use of lexical and structural information. Through several case studies with different types of diagrams and tasks, we show that our generic approach obtains better results than dedicated state-of-the-art algorithms, such as AURA, PLTSDiff or UMLDiff, on the exact same datasets used to introduce (and evaluate) these algorithms.
\end{abstract}

Index Terms-Diagram Differencing, Search-Based Software Engineering, Approximate Graph matching, Identifier splitting.

\section{INTRODUCTION}

\subsection{Context}

Most software engineering activities lead to the production of software artifacts, which help describe the functionalities, architectures, and designs of software systems. These artifacts are generally represented as diagrams consisting of entities linked by relationships and they are used to describe the structure or behavior of a software system. For a given system, some diagrams (e.g., class diagrams) will inform about its structure (or static view) by focusing on components and their inter-connections while others (e.g., sequence diagrams or labeled transition systems) will capture its behavioral description (or dynamic view). Comparing such software artifacts is, especially in maintenance/evolution, a recurrent task for which researchers and practitioners need efficient algorithms and tools. More specifically, given two diagrams generated by software activities, there is interest in retrieving the similarities and differences between them. Fulfilling this type of interest typically involves the matching of elements (entities or relations) of one diagram to elements of the other; after which the sought similarities and differences can be inferred.

\subsection{Problem}

There are many ways to define how the elements of two diagrams can be matched. In the simplest case (one-to-one matching), we can consider that one entity in a given diagram cannot be matched to more than one entity in the other diagram. Most of the proposed research

M. Kpodjedo, Galinier, Antoniol, and Guéhéneuc are with the Ecole Polytechnique of Montreal.

M. Ricca is with the Universita di Genova. work abides to this restriction, which works well in a number of settings. However, from one diagram to another, there can be multiple correspondences; for example, several entities of one diagram may be merged into a single entity in the other diagram.

In software engineering, research literature on diagram matching is mostly focused on source code and class diagrams [1], [2], [12], [18], [28], [30], [31]. Other related problems include the matching of sequence diagrams [22] or labeled transition systems [4]. The analysis of existing literature reveals in most cases an inability to simultaneously address three main concerns: genericness, accuracy, scalability.

Genericness refers to the adaptability of a given algorithm to a large variety of related problems. This concern is actually an important limitation of most of the proposed works, which only address a specific problem and are tailored for a specific representation. Only few works, such as [15], [8], [29], propose generic matching approaches applicable to different problems and diagram representations. However, these generic proposals often have limitations of their own regarding their scalability or the accuracy of the returned solutions. For example, GenericDiff [29] is less accurate than UMLDiff [30], [31], an earlier work (from the same authors) dedicated to the differencing of class diagrams while, regarding scalability, a recent work [15] cannot be used for very large diagrams.

\subsection{Running Example}

Figure 1 presents two subsequent class diagrams $D_{1}$ and $D_{2}$ of a given system. Based on their names, some elements can be easily matched from one diagram to another: (Ticket, Ticket), (Lottery, Lottery), (Lottery.restart, Lottery.restart). However, these perfect matches are not enough for a complete assessment 

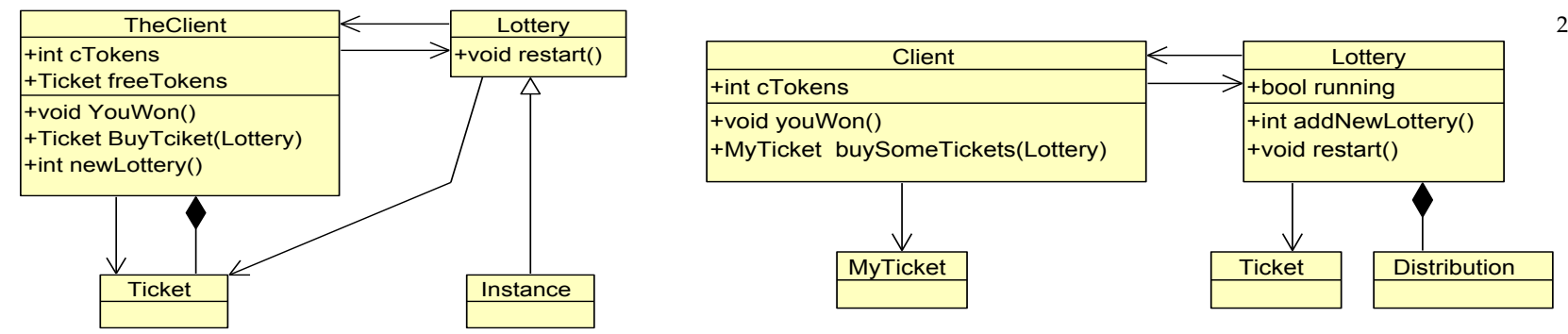

Figure 1. Our Running Example: Two Class Diagrams to be matched

of the correspondences between the two diagrams. In our example, the actual changes can be described as follows:

1) the class TheClient was renamed into Client;

2) the class Ticket was split into MyTicket and Ticket;

3) the method newLottery was moved from TheClient to Lottery and renamed addNewLottery;

4) the method BuyTciket (containing a typo) was renamed buySomeTickets;

5) the attribute cTokens was renamed cTickets;

6) the method YouWon was renamed youWon;

7) the class Instance was deleted;

8) a new class Distribution was added;

9) the attribute freeTokens was deleted;

10) a new attribute running was added (in Lottery).

Operations 1 to 6 establish imperfect, but correct, correspondences between the classes, methods and attributes, e.g., (\{TheClient $\},\{$ Client $\})$ or (\{Ticket $\}$, \{MyTicket, Ticket\}). Operations 7 to 10 are implied by these matches. These diverse operations reflect, between the two diagrams, differences to which one can assign certain likelihood or an implementation cost. In our approach, each possible difference is assigned a cost and the best matching is simply the one with the cheapest overall cost.

Such formulation is inspired from an approach in graph matching literature referred to as Error-Tolerant Graph Matching (ETGM) [5] [6] [24]. In a basic ETGM framework, matchings between graphs are one-to-one correspondences (a node is matched to at most one node) between the nodes of two graphs. A matching is represented by a set of node matches, which induces correspondences between arcs and determines which nodes and arcs are unmatched. Our proposal extends this framework by considering two additional aspects: lexical information (absent from basic graphs) and multiple correspondences (e.g., (\{Ticket $\}$, \{MyTicket, Ticket\})).

\subsection{Solution}

We propose MADMatch (Many-to-many Approximate Diagram Matching), a diagram matching approach based on a combinatorial optimization problem (COP) formulation. Our approach is built around three important components: (i) a simple and uniform formalism used to model a diagram as a labeled graph, (ii) a definition and valuation of the possible differences between two graphs to assess the quality of any given matching, and (iii) an advanced heuristic (tabu search) used to retrieve a matching of minimum cost.

To the best of our knowledge, MADMatch is the first approach for software diagram matching able to retrieve many-to-many correspondences. It does so by establishing one-to-one correspondences between sets of nodes (possibly singletons). Whenever a matched set contains more than one node, it is considered a merge. For example, given two possible correspondences $(x, y)$ and $(x, z)$ for a given node $x$, MADMatch allows the inclusion of both possibilities by merging $y$ and $z:(\{x\},\{y, z\})$. This feature improves genericness (by extending the range of problems that can be addressed) and accuracy (by reporting splits and merges), but adds to scalability issues (by enabling a much higher number of matching combinations). Interestingly, this latter concern can be significantly mitigated by a key difference between basic graphs and diagrams: the wealth of lexical information present in diagrams, which we use in innovative ways to reduce the number of possible combinations.

The present paper builds on our previous work on approximate graph matching, from theoretical experiments [17] to software engineering applications [18]. In particular, MADMatch is an extension of our previous work on the evolution of large objectoriented (OO) systems [18], which also featured a tabu search for a combinatorial optimization formulation. There are, however, many key differences and we claim four new contributions. First, we now address many-to-many matching. Second, we propose new search space reduction techniques using lexical and structural information. Third, we demonstrate the applicability of our approach on different categories of diagrams and include comparisons with state-ofthe art techniques: UMLDiff (design differencing) [30], [31], AURA (API evolution) [28], and PLTSDiff (labeled transition systems) [4]. Fourth, we propose a qualitative study with new insights on class diagram evolution. Our empirical study spans more than 80 versions from $4 \mathrm{OO}$ systems along with the replication of studies presented in reputable conferences (such as WCRE and ICSE) and is thus one of the biggest ever conducted on diagram matching for software engineering. Obtained results demonstrate that MADMatch contributes to the state-of-art with respect to 
genericness, scalability, and accuracy. MADMatch is publicly available as a web application ${ }^{1}$.

\subsection{Outline}

The remainder of this paper is organized as follows. Section 2 summarizes related work and introduces the approaches to which we compare MADMatch. We then present our approach by detailing how we model the diagram matching problem (Section 3), how we solve it (Section 4), and how we integrate lexical information in both the modeling and the solution (Section 5). Section 6 reports our empirical evaluation of MADMatch, which is mainly focused on OO design differencing but also includes matching scenarios such as API evolution, and the differencing of labeled transition systems and sequence diagrams. Section 7 proposes additional and more detailed discussion about MADMatch results and software evolution in general. Section 8 concludes and outlines future work. Finally, we provide in an appendix (Section 9) a detailed walkthrough of our algorithm.

\section{Related Work}

The comparison of software artifacts has been addressed in the research literature from many different perspectives, among which prominently software evolution. Many matching algorithms have been proposed to help developers in identifying structural changes between the designs of subsequent releases of large OO systems [1], [12], [18], [8], [27], [30], [31]. In a typical setting, a design representation, usually a class diagram, is first recovered from the code and then a matching algorithm is applied to pairs of versions to gain insight on the design evolution. Many techniques have also been proposed to help developers cope with the evolution of frameworks or Application Programming Interfaces (API).

We present in the following a selection of publications relevant to the present work.

\subsection{Design Differencing}

Antoniol et al. [1] presented one of the earliest approaches addressing the problem of detecting changes between the designs of subsequent releases of a given system. The approach recovers the design from the source code in an intermediate representation and compares it with subsequent releases by using a maximum match algorithm [7] applied to a bipartite graph. Nodes in the bipartite graph are the classes of the two releases and the similarity between them is derived from class and attribute/method names by means of string edit distance ${ }^{2}$.

1. http://tools.soccerlab.polymtl.ca/madmatch/index.php

2. The algorithm did not deal with the class relations.
More recently, Xing et al. [30], [31] proposed UMLDiff for the differencing of the UML class diagrams of subsequent versions of $\mathrm{OO}$ systems. The tool, implemented as an Eclipse plug-in with a PostgreSQL database, integrates a fact extractor that reverse-engineers, from Java source code, models of OO systems that include "classes, the information they may own, the services they can deliver, and the associations and relative organization among them". UMLDiff takes as input this model and identifies moves (e.g., an attribute is moved from one class to another) and renamings of elements. More specifically, given two versions of an $\mathrm{OO}$ software system and their reverse-engineered diagrams represented as two graphs, UMLDiff first retrieves perfect matches (entities having the same information in both graphs), which serve as landmarks used to recover the actual changes between the two versions. Using lexical and structural similarity between elements from the two graphs, UMLDiff proceeds to multiple rounds of matching, with each match between two elements serving as new evidence for the matching of other related elements. Two user-defined similarity thresholds control the matching algorithm: MoveThreshold for moves and RenameThreshold for renaming. UMLDiff also integrates a post-processing mechanism to identify behavior redistribution, which allows somewhat to retrieve some one-to-many correspondences. UMLDiff takes as input very detailed UML representations which improve its accuracy but hinders its genericness. Subsequent attempts by its authors to improve on this aspect result in the proposal of GenericDiff [29]. However, as reported in [29], GenericDiff is still a work in progress and is actually less accurate than UMLDiff. Thus, in this paper, we use UMLDiff for comparison purposes.

Genericness is a key concern and feature of the recent work proposed by Kimelman et al. [15]. To match architectural diagrams in an extended range of scenarios relevant to software engineering, the authors of [15] propose a generic Bayesian framework. Their approach includes a generic meta-model for diagrams accounting for syntactic, semantic, and even visual information. It tries to emulate human reasoning about correspondences and proposes a bayesian framework that integrates different evidence (label similarity, position similarity, etc.) in its matching process. A bayesian correspondence model is thus devised and used to formulate the problem of retrieving one-to-one, one-to-many, many-to-one correspondences; many-to-many correspondences are ruled out due to scalability issues. A greedy search is applied: the best correspondence is added at each iteration. The proposed algorithm AMMO (Automated Matching of Models) and a faster variation AMMO-Lite were applied with success to a range of different tasks. There are however some foreseeable scalability issues given that AMMO-Lite has a worst-case complexity 
of $O\left(N^{3} \log N\right)$ and requires as much as 6175 seconds to match diagrams of about 637 nodes (see Table 8 in [15]). Our attempts to obtain datasets from [15] were unsuccessful.

Another relevant work for design differencing is PLTSDiff, an approach proposed in [4] to compute the structural difference between State-based Models. PLTSDiff is dedicated to the differencing of partial labeled transition systems (PLTS). It is based on the computation and propagation of similarity scores assigned to pairs of states taken from the two PLTS to be matched. Such similarity scores are based on the networks of states and transitions surrounding the considered states. The technique is demonstrated on a small case study of three PLTS, which we reuse in this paper, for comparison.

In some other cases, design differencing algorithms are used as operators for model management activities, which are primarily about finding, in a database, possible correspondences for a given statechart. An example of such research line is [23], which proposes research work on specialized operators of model management referred to as Match and Merge. These operators are more sophisticated and specific than those targeted by our approach. For example, their Merge operator operates at the level of models whereas our merges are defined for nodes in a given diagram.

\subsection{API Evolution}

Several approaches have been proposed to address the Application Programming Interface (API) evolution problem [9], [14], [25], [28], [32]. The objective is to retrieve correspondences between elements (often methods) of different versions of a considered API. There are two main perspectives to succeed in this task. The first is to recover the evolution of the design between the two considered versions and then infer information about how specific calls should be modified; for example [32] uses the results of UMLDiff [30], [31] to provide such information. The second is to consider the elements separately and compute similarity measures to attach some level of likelihood to the fact that an element A1 from the old version has been replaced by element A2 [14], [25], [28], [9].

We chose for comparison purposes AURA [28], a recent proposal proven to outperform most of the previous approaches [14], [25]. AURA combines call dependency and text similarity analysis to retrieve the evolution of an API. It does not require any userdefined parameter and, according to its authors, is the first approach able to automatically handle one-tomany and many-to-one matchings. It is available as an Eclipse plug-in and its authors made their results available to us.

\section{Modeling Diagram Matching}

In our approach, we formulate diagram matching as an optimization problem, which solutions are the
Table 1

Modeling class diagrams

\begin{tabular}{|c|c|c|}
\hline \multicolumn{3}{|l|}{ Nodes } \\
\hline Type 0 & & subprograms and packages \\
\hline Type 1 & & classes and interfaces \\
\hline Type 2 & & methods \\
\hline Type 3 & & attributes \\
\hline Arcs & $A \rightarrow B$ & \\
\hline Type 1 & & class A "uses" class B \\
\hline Type 2 & & class A "aggregates" class B \\
\hline Type 3 & & class A inherits from class B \\
\hline Type 4 & & method A calls method B \\
\hline Type 5 & & method A uses attribute B \\
\hline Type 6 & & class B is the type of attribute A \\
\hline Type 7 & & class B is the return type of method A \\
\hline Type 8 & & class B is an input type of method A \\
\hline Type 9 & & entity A contains entity B \\
\hline
\end{tabular}

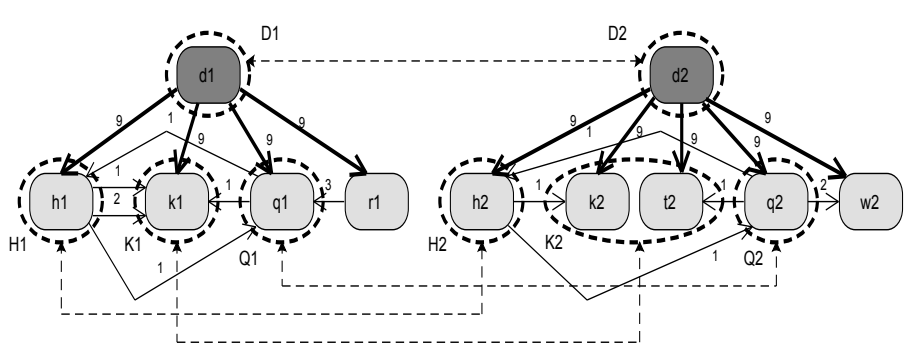

Figure 3. Example of a matching between two graphs

potential matchings between two input diagrams. Each potential solution (matching) is assigned a cost and the sought solution is the one with a minimum cost. In the following, we describe how we represent diagrams and matchings and then detail the different constituents of the proposed cost function.

\subsection{Diagram Representation}

We represent a diagram as a labeled multi-graph $D=(V, A)$, with a set $V$ of nodes and a set $A$ of arcs. Entities are represented by nodes, and relationships by arcs. Each node is labeled with a name, a type, and a list of features. Each arc has a type, with a special type (contains) accounting for the containment hierarchy found in many diagrams. In the following, we denote by $D_{1}=\left(V_{1}, A_{1}\right)$ and $D_{2}=\left(V_{2}, A_{2}\right)$ two input graphs to be matched.

Table 1 presents our modeling of class diagrams as graphs. When applied to our running example, it generates Figure 2. An extra node (root) is used to represent the main package while attributes and methods become nodes contained (arc of type 9) in a node (of type class). Possible features are modifiers (e.g., visibility), and types (for attributes, methods' output or inputs). To simplify reading and interpretation, we used different shades for each type of node and we did not display the node features.

\subsection{Matching Representation}

We represent in Figure 3 a matching between the subgraphs induced by the root nodes and classes from 


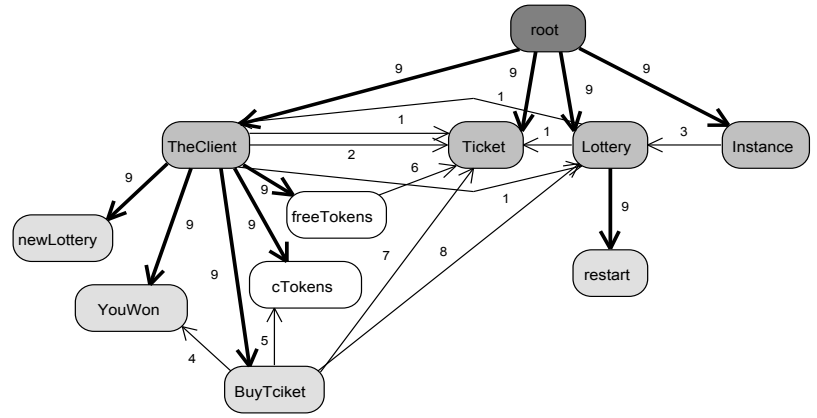

Figure 2. Graphs modeled from the running example

Figure 2. Disjoint sets from $V_{1}\left(\left\{d_{1}\right\},\left\{h_{1}\right\},\left\{k_{1}\right\},\left\{q_{1}\right\}\right)$ and $V_{2}\left(\left\{d_{2}\right\},\left\{h_{2}\right\},\left\{k_{2}, t_{2}\right\},\left\{q_{2}\right\}\right)$ are paired as follows: $\left(\left\{d_{1}\right\},\left\{d_{2}\right\}\right),\left(\left\{h_{1}\right\},\left\{h_{2}\right\}\right),\left(\left\{k_{1}\right\},\left\{k_{2}, t_{2}\right\}\right),\left(\left\{q_{1}\right\},\left\{q_{2}\right\}\right)$

Formally, a matching is a triple $\left(P_{1}, P_{2}, m\right)$ such that:

- $P_{1}$ is a family of subsets of nodes in $V_{1}$, and $P_{2}$ a family of subsets of nodes in $V_{2}$; and

- $m$ is a one-to-one correspondence between $P_{1}$ and $P_{2}$.

We refer to elements of $P_{1}$ (or $P_{2}$ ) as super-nodes and call node match an element of $m$ (a node match is thus a pair of super-nodes). We require the subsets in $P_{1}$ (or $P_{2}$ ) to be non-empty and two-by-two disjoint. In addition, only nodes of the same type can be merged in a super-node. The type of a super-node is the same as that of the nodes it is composed of. Its name is obtained by concatenating the names of these nodes. Its list of features is the union of the list of features from its components. In the following, we denote super-nodes with capital letters, as illustrated in Figure 3.

Given two nodes $x$ and $y$, we use the notation $\overrightarrow{x y}$ to represent the multi-set of arcs (relationships) from $x$ to $y$. In our example (see Figure 3 ), $\overrightarrow{h_{1} k_{1}}=\{1,2\}$ indicates that there is one arc of type 1 and another of type 2 from node $h_{1}$ to node $k_{1}$. This notation is extended to super-nodes as follows: $\overrightarrow{X Y}$ represents the multi-set of arcs whose starting point is a node in $X$ and whose endpoint is a node in $Y: \bigcup_{x \in X, y \in Y} \overrightarrow{x y}$. In our example, we have $\overrightarrow{\left\{d_{2}\right\}\left\{k_{2}, t_{2}\right\}}=\overrightarrow{d_{2} k_{2}} \cup \overrightarrow{d_{2} t_{2}}=$ $\{9,9\}$ (multi-set).

\subsection{Cost of a Matching}

We see a matching as a mathematical object that establishes direct correspondences between sets of nodes, which induce correspondences between sets of arcs. A good matching is one in which the matched elements (nodes or arcs) are as similar as possible. In a good matching, the discrepancies should be limited (and ideally avoided). Therefore, they will be penalized using a cost function. In addition, penalties will also be applied for nodes and arcs that are not involved in the matching.

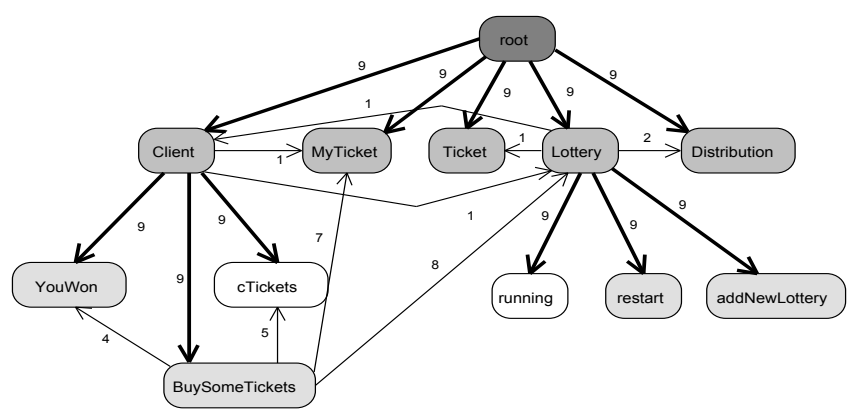

\subsubsection{Main Principles}

A matching establishes correspondences between sets of nodes. Every node match $\left(X_{1}, X_{2}\right) \in m$ pairs off a super-node $X_{1}$ in $D_{1}$ with a super-node $X_{2}$ in $D_{2}$. For example, in Figure 3, we have $\left(\left\{k_{1}\right\},\left\{k_{2}, t_{2}\right\}\right)$ also represented by $\left(K_{1}, K_{2}\right)$. Discrepancies between supernodes are computed using their names and features. To do so, we propose a function nodeDiff $\left(X_{1}, X_{2}\right)$ based on text similarity measures and weights for names (nameWeight) and features (featWeight); details are provided in Section 5.

Matched super-nodes induce correspondences between sets of arcs. An arc is involved in the matching if its two endpoint nodes are. More precisely, every pair of node matches $\left(\left(X_{1}, X_{2}\right),\left(Y_{1}, Y_{2}\right)\right) \in m \times m$ induces a correspondence between $\overrightarrow{X_{1} Y_{1}}$ (in diagram $D_{1}$ ) and $\overrightarrow{X_{2} Y_{2}}$ (in diagram $D_{2}$ ). The discrepancies between $\overrightarrow{X_{1} Y_{1}}$ and $\overrightarrow{X_{2} Y_{2}}$ are expressed by (i) the number $\left|\overrightarrow{X_{1} Y_{1}}-\overrightarrow{X_{2} Y_{2}}\right|$ of arcs present in $\vec{X}_{1} Y_{1}$ and not in $\overrightarrow{X_{2} Y_{2}}$, and (ii) the number $\left|\overrightarrow{X_{2} Y_{2}}-\overrightarrow{X_{1} Y_{1}}\right|$ of arcs present in $\overrightarrow{X_{2} Y_{2}}$ and not in $\overrightarrow{X_{1} Y_{1}}$. For example, when we consider in Figure 3 the pair of matches $\left(\left(H_{1}, H_{2}\right),\left(K_{1}, K_{2}\right)\right) \in m \times m$, we have $\mid \overrightarrow{H_{1} K_{1}}-$ $\overrightarrow{W_{2} Y_{2}}|=|\{2\} \mid=1$. If we consider the pair of matches $\left(\left(D_{1}, D_{2}\right),\left(H_{1}, H_{2}\right)\right) \in m \times m$, there are no discrepancies as $\overrightarrow{D_{1} H_{1}}=\overrightarrow{D_{2} H_{2}}=\{9\}$.

A matching is not required to establish correspondences for all nodes. Thus, some nodes will be unmatched and all their adjacent arcs will be considered unmatched as well. There is a penalty for every unmatched node and every unmatched arc. In our example, there are two unmatched nodes $\left(r_{1}\right.$ and $\left.w_{2}\right)$ and all the arcs adjacent to these $\left(\overrightarrow{d_{1} r_{1}}, \overrightarrow{r_{1} q_{1}}, \overrightarrow{d_{2} w_{1}}\right.$, $\overrightarrow{w_{2} q_{2}}$ ) are unmatched arcs.

Finally, we impose, through a simple and uniform mechanism, additional penalties for merges (supernodes with a cardinality above 1). Every time a penalty involves a super-node, this penalty is multiplied by the cardinality of this super-node. In particular, (i) the discrepancy value between two supernodes $X_{1}$ and $X_{2}$ (nodeDiff $\left(X_{1}, X_{2}\right)$ ) is multiplied by $\left|X_{1}\right| \times\left|X_{2}\right|$, and (ii) whenever a given arc $\overrightarrow{X_{i} Y_{i}}$ is not perfectly matched, the penalty is multiplied by $\left|X_{i}\right| \times\left|Y_{i}\right|$. 
Table 2

Basic Cost parameters

\begin{tabular}{l|l}
\hline & Description \\
\hline$C_{n+}$ & maximum cost of a label match between two nodes \\
\hline Weight & nameWeight, featWeight weight names and features \\
\hline$C_{n-}^{1}$ & cost for an unmatched node in the first graph \\
\hline$C_{n-}^{2}$ & cost for an unmatched node in the second graph \\
\hline$C_{a+}^{1}$ & cost for each arc match error in the first graph \\
\hline$C_{a+}^{2}$ & cost for each arc match error in the second graph \\
\hline$C_{a-}^{1}$ & cost for each unmatched arc in the first graph \\
\hline$C_{a-}^{2}$ & cost for each unmatched arc in the second graph \\
\hline
\end{tabular}

\subsubsection{Formal Definition}

We denote by $f$ the cost of a matching $\left(P_{1}, P_{2}, m\right)$. The sets of the nodes and arcs involved in a matching are denoted by $V_{+}^{1}$ and $V_{+}^{2}$ (nodes) and $A_{+}^{1}$ and $A_{+}^{2}$ (arcs); the sets of unmatched nodes and arcs are denoted by $V_{-}^{1}$ and $V_{-}^{2}$ and by $A_{-}^{1}$ and $A_{-}^{2}$. The value of $f$ is the sum of terms that penalize the discrepancies between matched nodes $\left(f_{n+}\right)$ and matched arcs $\left(f_{a+}^{1}\right.$ and $\left.f_{a+}^{2}\right)$, and the presence of unmatched nodes $\left(f_{n-}\right)$ and unmatched arcs $\left(f_{a-}^{1}\right.$ and $\left.f_{a-}^{2}\right)$. Note in addition that $f$ depends on a set of cost parameters whose roles are described in Table 2. The cost function is defined by the following equations:

- $f=f_{n+}+f_{a+}^{1}+f_{a+}^{2}+f_{n-}+f_{a-}^{1}+f_{a-}^{2}$

- $f_{n+}=C_{n+} \sum_{\left(X_{1}, X_{2}\right) \in m}\left|X_{1}\right|\left|X_{2}\right| . \operatorname{nodeDiff}\left(X_{1}, X_{2}\right)$

- $f_{a+}^{1}=C_{a+}^{1} \cdot \sum_{\left(\left(X_{1}, X_{2}\right),\left(Y_{1}, Y_{2}\right)\right) \in m \times m}\left|X_{1}\right| .\left|Y_{1}\right|$. $\left|\overrightarrow{X_{1} Y_{1}}-\overrightarrow{X_{2} Y_{2}}\right|$

- $f_{a+}^{2}=C_{a+}^{2} \cdot \sum_{\left(\left(X_{1}, X_{2}\right),\left(Y_{1}, Y_{2}\right)\right) \in m \times m}\left|X_{2}\right| \cdot\left|Y_{2}\right|$. $\left|\overrightarrow{X_{2} Y_{2}}-\overrightarrow{X_{1} Y_{1}}\right|$

- $f_{n-}=C_{n-}^{1} \cdot\left|V_{-}^{1}\right|+C_{n-}^{2} \cdot \mid V_{-}^{2}$

- $f_{a-}^{1}=C_{a-}^{1} \cdot \sum_{\left(x_{1}, y_{1}\right) \in A^{1}} w\left(x_{1}\right) \cdot w\left(y_{1}\right)$

- $f_{a-}^{2}=C_{a-}^{2} \cdot \sum_{\left(x_{2}, y_{2}\right) \in A_{-}^{2}} w\left(x_{2}\right) \cdot w\left(y_{2}\right)$

where $w(x)=1$ if $x$ is unmatched or $w(x)=|X|$, with $X$ the super-node that contains $x$.

\subsubsection{Tuning of the Cost Model}

Taken individually, the parameters presented in Table 2 do not provide insights as to the kind of matching they would induce. We thus propose a reduced and user-friendly set of ratios that simplify a deliberate tuning of these cost parameters. The goal of this new set of parameters is to help users in the setting of meaningful aspects such as the level of tolerance to discrepancies, the weighting of different kinds of information (e.g., lexical vs. structural).

We define five aggregate parameters (see Table 3) which help specify the sought matchings:

1) dropNodeRatio and dropArcRatio calibrate error tolerance respectively to lexical dissimilarity and structural differences, the lower those values the less tolerant the matching.

2) arcWeight and nameWeight calibrate the importance of different sources of information, respectively structural information and the weighting of the entities' names.
Table 3

Aggregate cost parameters

\begin{tabular}{|c|c|c|}
\hline Parameters & \multicolumn{2}{|c|}{ Description } \\
\hline \multirow[t]{2}{*}{ dropNodeRatio } & Role & considering only nodes, drop or match? \\
\hline & Formula & $d n r=\frac{C_{n-}^{1}+C_{n-}^{2}}{C_{n+}}$ \\
\hline$(d n r)$ & Range & $\begin{array}{l}\text { Min=0: zero-tolerance on lexical changes; } \\
\text { Max=1: no penalty for node dissimilarity }\end{array}$ \\
\hline \multirow[t]{3}{*}{ dropArcRatio } & Role & considering only arcs, drop or match? \\
\hline & Formula & $d a r=\frac{C_{a-}^{1}+C_{a-}^{2}}{C_{a+}^{1}+C_{a+}^{2}}$ \\
\hline & Range & $\begin{array}{l}\text { Min=0: zero-tolerance on arc changes; } \\
\text { Max=1: no penalty for arc changes }\end{array}$ \\
\hline \multirow[t]{3}{*}{ arcWeight } & Role & arc information over node information? \\
\hline & Formula & $a w=\frac{C_{a+}^{1}+C_{a+}^{2}}{C_{n+}}$ \\
\hline & Range & $\begin{array}{l}\text { Min=0: arc information is not used; } \\
\text { Max } \rightarrow \infty \text { : only arc information is used }\end{array}$ \\
\hline \multirow[t]{3}{*}{ asymmetry } & Role & additions over deletions? \\
\hline & Formula & $a s y=\frac{C_{n-}^{2}}{C_{n-}^{1}}=\frac{C_{a-}^{2}}{C_{a-}^{1}}=\frac{C_{a+}^{2}}{C_{a+}^{1}}$ \\
\hline & Range & $\begin{array}{l}\text { Min=0: additions are penalty-free; } \\
\operatorname{Max} \rightarrow \infty \text { : deletions are penalty-free }\end{array}$ \\
\hline \multirow[t]{3}{*}{ nameWeight } & Role & node name over features? \\
\hline & Formula & nameW eight $=1-$ featWeight \\
\hline & Range & $\begin{array}{l}\text { Min=0: names are irrelevant; } \\
\operatorname{Max}=1 \text { : Feature information is irrelevant }\end{array}$ \\
\hline
\end{tabular}

3) asymmetry is defined to take into account the direction of the matching.

For example, if we set dropNodeRatio $=0$ and dropArcRatio $=0$, the sought matching is one which does not tolerate any discrepancies between both matched nodes and matched arcs. Note that given aggregate parameters, we can recompute the values of each of the basic parameters presented in Table 2 (see Appendix for details).

\section{Searching For a Good Matching}

In our formulation of diagram matching, finding an optimal solution is an NP-hard problem [26]. Therefore, if $P \neq N P$, the only algorithms that can find optimal solutions have an exponential worst-case complexity, i.e., they require prohibitive computation times even for medium-size graphs. We resort to meta-heuristics, in particular Tabu Search [11], to obtain near optimal solutions in acceptable computation times. The algorithm we propose has a worst case complexity ${ }^{3}$ of $O\left(n^{2}\right)$, with $\mathrm{n}$ being the number of entities in one diagram, and consists in a tabu search enhanced by various mechanisms.

Our tabu search algorithm explores the set of possible matchings and tries to find one having a minimum cost. As depicted in Figure 4, it first generates an initial matching between nodes that share the same ancestry (with respect to the containment tree defined in Section 3.1), name, and features. Then, to enable greater scalability, it proceeds to a reduction of the search space. The actual search follows and for each iteration, the algorithm generates a set of neighboring solutions (matchings) and computes their cost. The best configuration is chosen, while avoiding returning

3. Details are provided in [16] 


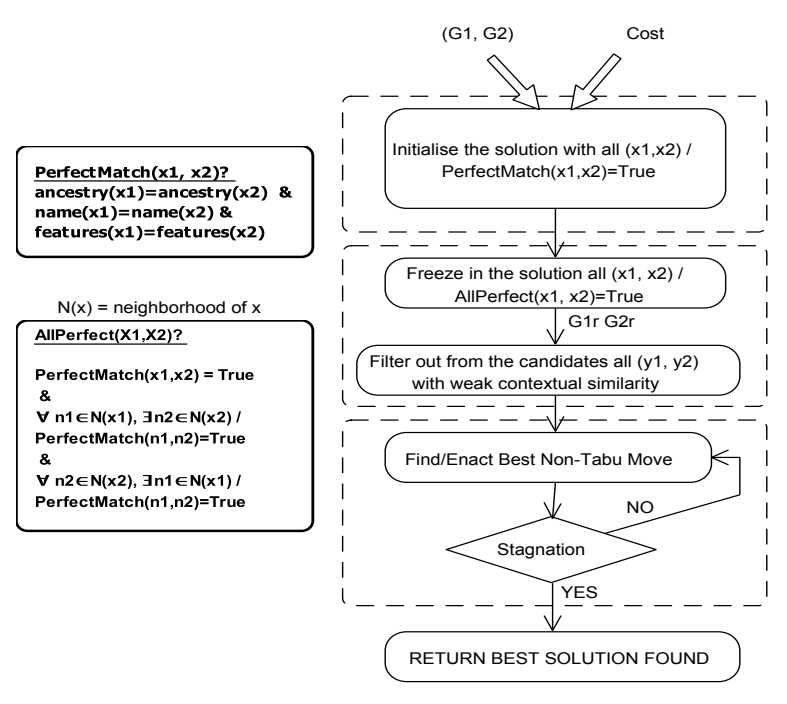

Figure 4. Block diagram of the MADMatch algorithm

toward solutions recently visited by using a shortterm tabu list. When, after a certain number of iterations, there is no longer improvement with respect to the best solution found so far, the search is stopped and the solution with the minimal cost is returned.

We provide in the following subsections details on the above mechanisms.

\subsection{Reduction of the Search Space}

The search space (number of possible solutions) for a diagram matching problem can be very large and severely limit the scalability of proposed techniques. The problem has been previously mentioned in many related papers, such as [15] or [31], and addressed with mixed success. For example, applied to our running example, UMLDiff [31] cannot retrieve a match such as (TheClient.newLottery $\rightarrow$ Lottery.addNewLottery) because this evolution is the result of a move (newLottery moved from TheClient to Lottery) followed by a renaming (newLottery renamed to addNewLottery). Ignoring these types of matches (move + renaming) is a deliberate choice made by UMLDiff authors [31] to limit the number of possible combinations; the same goes for [15] which consider one-to-many and manyto-one matchings but not many-to-many matchings.

To address scalability issues, we propose a two-step reduction of the search space.

\subsubsection{Freezing parts of the initial solution}

There are often many perfect node matches between two diagrams considered for a matching. When such nodes also have perfectly identical neighborhoods (i.e., all the neighbors of the first node are perfectly matched to all the neighbors of the second node), we consider them as definitive matches (see Figure 4 for more details). These entities are irrelevant for the matching process because they interact only with entities already perfectly matched and cannot affect the matching of other entities.

In our example (see Figure 2), the nodes Ticket, Lottery, and restart of the first diagram will thus be matched to their counterparts (the nodes sharing the same ancestry and name) in the second diagram. Furthermore, the nodes named restart (in both diagrams) will be considered definitively matched because their respective neighbors are also perfectly matched (Lottery matched to Lottery). Consequently, no other node will be considered as a possible match for either of the nodes restart, thus reducing the number of possible combinations. The output of this filter consists in three sets: (i) a set of frozen perfect node matches, with nodes deemed irrelevant for the matching process, (ii) a set of perfect node matches (with non-perfect neighborhood matches) which can effectively inform and affect the matching process, and (iii) the sets of unmatched entities from both diagrams.

Nodes that are not involved in a frozen node match induce the subgraphs that are effectively used in our matching approach. In the following, we refer to these subgraphs as the relevant graphs $\left(G_{1 r}\right.$ and $\left.G_{2 r}\right)$.

\subsubsection{Discarding unlikely matches}

First, we assume that nodes of different types should be considered as impossible matches: for example, in a class diagram, it would hardly make sense trying to match an attribute to a package. The same assumption is used in other approaches, such as [30], [31].

Second, we filter out node matches that we deem unlikely. To achieve this goal, we propose the computation of a contextual similarity between two nodes based on a comparison of the textual information surrounding these nodes. Pairs of nodes whose contextual similarity values fail to reach a certain threshold are discarded and no longer considered in the search for a good matching. Details on the computation of our contextual similarity are provided in Section 5.2.

\subsection{Neighborhood and tabu mechanisms}

We described in the previous subsections how we generate an initial solution for our matching and how we reduce the search space that our tabu search [11] must explore. Starting from this initial solution, our tabu search algorithm moves iteratively from the current solution to a neighboring one. At each iteration, the algorithm chooses the best neighbor, i.e., the one with the best cost. Our tabu lists forbid recently-inserted node matches to leave the solution for a given number of iterations and recently-removed node matches to re-enter the solution for a given number of iterations.

In MADMatch, a move applied to a current solution consists in:

(a) adding a pair of previously unmatched nodes;

(b) removing a pair of nodes from the solution;

(c) adding a new node to a super-node; 
(d) removing a node from a super-node.

Using the moves (a), (b), (c), (d), MADMatch gradually builds and-or improves a solution. In particular, the moves (c) enable multiple correspondences through the addition of one node to an existing supernode and can be rolled back using the moves (d), which allow the removal of a node from a super-node. Readers are referred to the appendix (Section 9) for a detailed application of MADMatch to our running example.

\section{AdDRESSING LEXICAL INFORMATION}

In this section, we present our handling of lexical information in the diagrams. Our formulation of diagram matching is inspired from graph matching approaches with no specific details about textual information. The wealth of text found in diagrams is obviously a "gift", but one that has to be handled carefully. It presents enormous opportunities for the initialisation of a solution, the evaluation of the differences between nodes, and the pruning of the search space. These opportunities come with some challenges. The main one is the renaming of entities: entities' names are often composite strings obtained by the concatenation of basic terms, which can be words, acronyms, abbreviations, etc. From one diagram to another, these terms can be replaced, altered, etc. Consistent with this reality, our handling of textual information is based on the use of those basic units of text, which we recover with the Camel Case [3] split ${ }^{4}$.

Terms extracted from entities' names and features are used to compute the similarity metrics previously introduced in Sections 3 and 4. In the following, we detail how, given two nodes, we measure their discrepancies (nodeDiff) or their likelihood of being matched (contextual similarity).

\subsection{Node discrepancies}

We compute the difference between two nodes (nodeDiff) using textual similarity between their names and features. First, we apply identifier splitting on the strings and then compare the sets of terms obtained from the splitting.

\subsubsection{Similarity between sets of terms}

The comparison between two terms can be binary (are the terms equal?) or quantitative (how similar are the terms?).

The binary option can be used to retrieve the cardinality of the intersection between two sets of terms. For example, once the term splitting done on the names of the methods BuyTciket (assuming a typo) and buySomeTickets, we can easily and quickly compute

4. Note that more sophisticated identifier splitting techniques have been proposed but the Camel Case split remains the fastest and most widely used.
Table 4

Comparing "BuyTciket" and "buySomeTickets"

\begin{tabular}{l|l|l|l}
\hline$|L C S|$ & buy & some & tickets \\
\hline buy & $\mathbf{3}$ & 0 & 0 \\
tciket & 0 & 1 & $\mathbf{3}$ \\
\hline
\end{tabular}

that these two identifiers share one term (buy). A first (Boolean) similarity measure based on this option is:

$\operatorname{textSim}_{1}\left(\operatorname{str}_{1}, \operatorname{str}_{2}\right)=\frac{2 \times\left|\operatorname{splits}\left(\operatorname{str}_{1}\right) \cap \operatorname{splits}\left(\operatorname{str}_{2}\right)\right|}{\left|\operatorname{splits}\left(\operatorname{str}_{1}\right)\right|+\left|\operatorname{splits}\left(\operatorname{str}_{2}\right)\right|}$

where $\operatorname{splits}(X)$ represent the set of terms obtained from a string $X$.

For the quantitative option, we proceed to pairwise comparisons between all the terms of the first string and all the terms of the second string. Such comparisons are made using the Longest Common Substring (LCS), which, given two strings, returns the longest string that is a substring of both strings.

Table 4 illustrates the proposal with the strings BuyTciket and buySomeTickets. In the example, we have:

$\mid L C S($ buy, buy $)|=3| L C S,($ tciket, tickets $)|=|$ ket $\mid=3$

In this view, computing the similarity value of two strings equates to finding the best matching between the terms extracted from them. Such task can be modeled as an assignment problem and optimally solved by the Hungarian algorithm [19]. On the example displayed in Table 4, the result of the application of the Hungarian algorithm would be buy $\leftrightarrow$ buy and tciket $\leftrightarrow$ tickets, which means that BuyTciket (9 characters) and buySomeTickets (14 characters) share 6 characters.

A second (quantitative) textual similarity measure is computed as follows:

$\operatorname{textSim}_{2}\left(\operatorname{str}_{1}, \operatorname{str}_{2}\right)=\frac{2 \times \text { length }(\text { optim_term_match })}{\text { length }\left(\operatorname{str}_{1}\right)+\text { length }\left(\operatorname{str}_{2}\right)}$

where length $(X)$ is the number of characters of the string $X$ and optim_term_match is based on the output of the underlying assignment problem.

\subsubsection{Names and features' similarity}

Using Camel Case Splitting, we generate for nodes, sets of terms from their name (nameSet) and features (featList). Given two nodes, we use these sets of terms to compute similarity values for names (or features).

For features of a node, we opted ${ }^{5}$ for the first similarity measure textSim $_{1}$.

$$
\text { featSim } \rightarrow \text { text }_{\text {Sim }}
$$

5. Preliminary experiments on a sample of $10 \%$ of the version pairs used in Section 6 show that using textSim 1 to determine features similarity is about $1 / 8$ faster than using textSim 2 and without any notable accuracy differences. 
In contrast, the name of a node generally consists of a few, generally significant, terms. We thus deemed the use of textSim 2 both indicated and viable. Moreover, to mitigate the fact that terms can have different lengths, we also use textSim 1 to avoid situations in which the length of some terms completely bias the computed similarity. For example, consider the names supremeFarOut and extraordinaryFarOut; both supreme and extraordinary contain more characters than the two terms far and out and could significantly lower the similarity between supremeFarOut and extraordinaryFarOut. We thus define the text similarity between two names as follows:

$$
\text { nameSim } \rightarrow \max \left(\text { textSim }_{1}, \text { textSim }_{2}\right)
$$

The similarity between two nodes is computed as follows: nodeSim $=$ nameWeight $\times$ nameSim + featWeight $\times$ featSim (with nameWeight, and featWeight the respective weights for names and features). Their discrepancies are expressed by nodeDiff $=1$ - nodeSim, which is used in the cost computation of a node match (see Appendix for details).

\subsection{Contextual similarity}

Before the actual search for a good matching, we discard unlikely node matches based on an analysis of the involved diagram entities and their neighborhood. This contextual similarity is a measure of how close these entities are when taking into account the entities they interact with. In the following, we detail how we capture and represent the context of an entity, then propose a computation of a similarity measure.

\subsubsection{Computing the "context" of an entity}

From the splitting of entities' names into terms, we propose the computation of an entity "context", which informs about terms present in its neighborhood.

Unlike the standard approach in software traceability [20] in which a document is defined only by the terms that it contains, we also consider, for a given entity $e$, the terms contained in the neighbors of $e$ (i.e., the entities with arcs coming to or from $e$ ). Robustness to renaming is the main goal of our termal context. For a given entity, we assume that a renaming can occur but is unlikely to affect all the terms contained in the entity name. The same goes for the neighbors of that entity. Given two entities that correspond to a correct match, we assume that the chances that their termal contexts do not share common terms are quite slim.

More formally, a relation between an entity ent and a term trm is defined by a triplet $(i, f, t)$ where $i$ is the number of occurrences of trm in the entity name, $f$ is the number of occurrences of $t r m$ in entities with an arc going to ent (in-neighbors), and $t$ is the number of occurrences of trm in entities with an arc coming from ent (out-neighbors). A single variable $S$ (defined as the sum of $i, f$, and $t$ ) can be used to capture the size of the relation between an entity and a term. The termal context of an entity represents its relations with terms present in its name and its neighborhood. Its size is the sum of the sizes of those relations. The relation of an entity with a given term can be further refined if the type of the neighbors is taken into account ${ }^{6}$. Figure 5 illustrates such a relation, using the entity TheClient and the term lottery. The relation between the entity TheClient and the term lottery is featured at the center of the figure. The entity (class) TheClient has both an out-relation and an in-relation with the entity (class) Lottery. Plus, it contains the entity (method) newLottery. Thus, the termal context of TheClient relatively to the term lottery is represented by the triplet $(0,1,2)$, meaning that the entity name does not contain the term, but has one (1) in-neighbor whose name contains lottery (the class Lottery) and two out-neighbors whose names contain lottery (the class Lottery and the method newLottery).

Similarly to a common practice in information retrieval where researchers define a document-term matrix [20] from which they can infer similarity between documents, we generate entity-term matrices (ETM) that contain the termal contexts of entities. In our case, ETM are 3-dimension matrices used to represent the relation of entities to terms: the first dimension represents the entities, the second dimension the terms and the third the different counters expressing occurrences of the terms within the names of the entity itself or its neighbors. For a given entity-term matrix ETM, $E T M[e][t][c]$ represents the number of occurrences of a term $t$ relatively to an entity $e$ and a variable $c$ (expressing either $i, f$, or $t$ ). In particular, ETM[e][t][0] is the number of occurrences of the term $t$ in the name of the entity $e$.

\subsubsection{Computing the contextual similarity}

Once we generate entity-term matrices of the two considered diagrams, we used them to compute contextual similarity values between entities. Given that we consider many-to-many matching, an entity from one graph may be matched to several entities from the other graph. Thus, with respect to similarity measures, we prefer inclusion to equality: considering two entities $e_{1}$ and $e_{2}$, the interesting question is how much of the termal context of $e_{1}\left(T F_{1}\right)$ can be retrieved in that of $e_{2}\left(T F_{2}\right)$ and vice versa. We thus define the contextual similarity of $e_{1}$ relatively to $e_{2}\left(\operatorname{cSim}_{1}\right)$ and that of $e_{2}$ relatively to $e_{1}\left(\mathrm{cSim}_{2}\right)$.

To do so, we first determine the intersection $\left(T C_{1} \cap\right.$ $T C_{2}$ ) of the termal contexts, computed as follows:

$$
\sum_{t \in T_{1} \cap T_{2}} \sum_{i=0 . . k} \min \left(E T M_{1}\left[e_{1}\right][t][i], E T M_{2}\left[e_{2}\right][t][i]\right)
$$

6. Another option could be to consider the types of the arcs linking the entities but the type of an entity is much more stable information. 


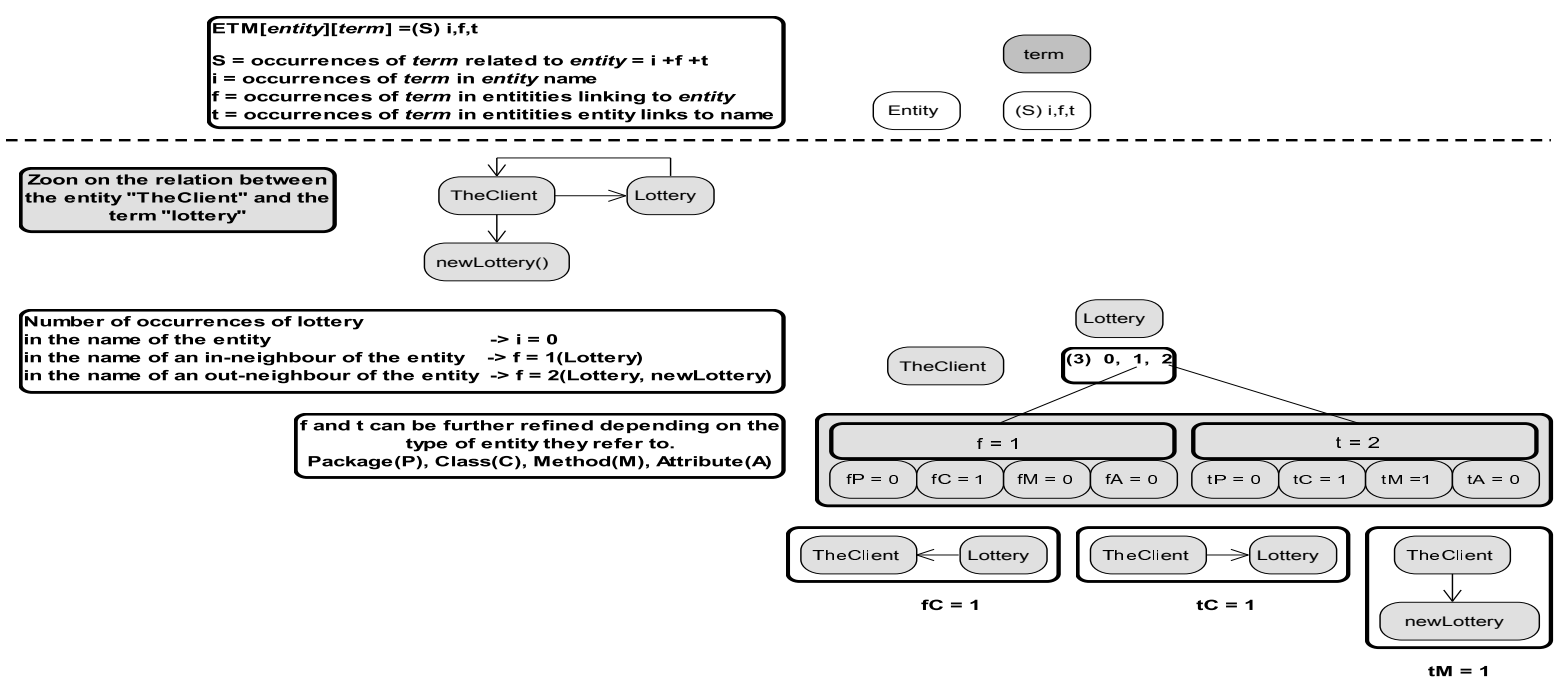

Figure 5. Samples from the entity-term matrices of the running example

with $T_{1}$ and $T_{2}$ the sets of terms recovered from the two diagrams, ETM $M_{1}$ and $E T M_{2}$ the entity-term matrices of the two diagrams, and $k$ the number of different term counters (typically, three for the triplet $(\mathrm{i}, \mathrm{f}, \mathrm{t})$ defined in 5.2.1).

Then, we compute the contextual similarity values $c \operatorname{Sim}_{1}$ and $\mathrm{cSim}_{2}$

$$
c \operatorname{Sim}_{1}=\frac{\left|T C_{1} \cap T C_{2}\right|}{\left|T C_{1}\right|}, \quad c \operatorname{Sim}_{2}=\frac{\left|T C_{1} \cap T C_{2}\right|}{\left|T C_{2}\right|}
$$

These values are used to discard pairs of entities for which both $c_{\text {Sim }}$ and $c_{\text {Sim }}$ are under a given threshold. In MADMatch, we chose 0.5 as this threshold, which means that pairs of entities are considered only if at least half of the termal context of one is contained in the termal context of the other.

\section{MADMATCH EMPIRICAL EVALUATION}

The main goal of our empirical evaluation is to investigate the accuracy of our approach, its scalability, and its applicability in different diagram matching scenarios. A preliminary phase for such investigation is the collection of relevant datasets. Unfortunately, to the best of our knowledge, there is no repository of software diagrams readily usable for our experiments. In addition, as we noted in Section 2, our attempts to obtain industrial datasets from the study presented in [15] were ultimately unsuccessful, due to confidentiality issues. The creation of a diverse and large benchmark is outside the scope of our work. Thus, we chose to focus on OO design artifacts, which are (i) possibly the most studied objects for diagram differencing, and also (ii) the artifacts for which we have a customized reverse-engineering tool.

Our empirical study on OO design differencing spans 4 systems and more than 80 releases. It is one of the biggest ever conducted on this topic and allowed us to investigate questions related to our approach accuracy and scalability. The applicability of MADMatch in different scenarios is then evaluated with a few other small case studies, taken mostly from recent research work published in reputable conferences such as ICSE and WCRE. The selected scenarios include API evolution, and the differencing of Labeled Transition Systems (LTS) and Sequence Diagrams (SD). They illustrate how MADMatch can be applied to other problems and the kind of results and insights its application can provide. Except for SDs, each matching scenario is assessed using a compared evaluation of MADMatch with another specialized technique.

We report in the following the evaluation of MADMatch for OO design differencing and other matching scenarios. We first report the settings of MADMatch and our procedure for compared evaluations, then present the different case studies. Note that, out of space considerations, we only reported key results and we refer the reader to [16] for more details.

\subsection{Settings and Procedures}

We present in this section details about the settings of MADMatch and the general procedure we applied for our case studies.

\subsubsection{MADMatch settings}

With respect to the local search, we set the stagnation number at 100, which means that MADMatch stops if after 100 moves there is no improvement on the best recorded solution. Recently-inserted node matches are forbidden to leave the solution for a number of it- 
erations randomly chosen between 5 and $10^{7}$ while recently-removed node matches are forbidden to reenter the solution for a number of iterations randomly chosen between 10 and 20. Our algorithm, like most meta-heuristics, is stochastic and there is no guarantee of obtaining identical solutions for different runs. To mitigate this issue, when moves of the same cost are considered, the first (using entities' assigned numbers) one is always selected.

Regarding the cost model, the default values of the aggregate parameters are set as follows:

- dropNodeRatio $=0.7 \rightarrow$ for a high tolerance to text dissimilarity

- dropArcRatio $=0.7 \rightarrow$ for a high tolerance to structure dissimilarity

- arcWeight $=0.2 \rightarrow$ information brought by one relationship is about $20 \%$ of information brought by one node

- nameWeight $=0.5 \rightarrow$ the entity name counts for half the text similarity between two entities

- asymmetry $=1 \rightarrow$ the matching direction is not taken into account

The above setting is inspired from previous experiments that we conducted on class diagrams [18] in a one-to-one matching context.

All computations were performed on a dual Opteron server $(3 \mathrm{GHz})$, with 16GB of RAM, running RedHat Advanced Server.

\subsubsection{Compared Evaluation Procedure}

Given the size of some of the considered diagrams and the possibility of many-to-many matching, building an oracle is a prohibitive and uncertain task. Thus, to assess the accuracy of the solutions returned by MADMatch, we quantify the number of correct matches with respect to results from another technique.

\section{Differential accuracy measures}

For our accuracy measures, we consider only nonperfect node matches (as defined in Section 4). Given two sets of non-perfect node matches $M_{1}$ and $M_{2}{ }^{8}$ obtained from two different techniques, we define, relatively to a given matching, its percentage of agreement with the other matching set (pAgreement), its differential precision (dPrecision), and its differential recall (dRecall):

$$
\begin{aligned}
& \text { - } p \text { Agreement }\left(M_{1}\right)=\frac{\left|M_{1} \cap M_{2}\right|}{\left|M_{1}\right|} \\
& \text { - } \operatorname{pAgreement}\left(M_{2}\right)=\frac{\left|M_{1} \cap M_{2}\right|}{\left|M_{2}\right|} \\
& \text { - } M_{1 x}=M_{1}-M_{2}, M_{2 x}=M_{2}-M_{1} \\
& \text { - } d \text { Precision }\left(M_{1}\right)=\frac{\left|\operatorname{correct}\left(M_{1 x}\right)\right|}{\left|M_{1 x}\right|} \\
& \text { - } d \text { Precision }\left(M_{2}\right)=\frac{\left|\operatorname{correct}\left(M_{2 x}\right)\right|}{\left|M_{2 x}\right|}
\end{aligned}
$$

7. Random selection of a number in a given interval is a wellknown technique aimed at further preventing cycling during a local search.

8. To simplify the comparison, multiple correspondences generate many one-to-one correspondences; for example, $(\{a, b\}\{c, d\})$ becomes $(\{a\},\{c\}),(\{a\},\{d\}),(\{b\},\{c\}),(\{b\},\{d\})$

$$
\begin{aligned}
& \text { - } d \operatorname{Recall}\left(M_{1}\right)=\frac{\left|\operatorname{correct}\left(M_{1 x}\right)\right|}{\left|\operatorname{correct}\left(M_{1 x} \cup M_{2 x}\right)\right|} \\
& \text { - } d \operatorname{Recall}\left(M_{2}\right)=\frac{\left|\operatorname{correct}\left(M_{2 x}\right)\right|}{\left|\operatorname{correct}\left(M_{1 x} \cup M_{2 x}\right)\right|}
\end{aligned}
$$

With these measures, we can assess whether one algorithm is better than the other by measuring their differences and evaluating which of the algorithm has less noise to filter out (dPrecision) and which one returns more correct node matches (dRecall). Given our definition, $d \operatorname{Recall}\left(M_{1}\right)=100-d \operatorname{Recall}\left(M_{2}\right)$ and thus the differential recall is a measure of the number of new node matches brought by one technique relatively to the other.

\section{Manual validation}

To retrieve the differential measures, we conducted extensive manual validation on the sets of (nonperfect) node matches exclusive to a given algorithm. To ease this task, MADMatch produces detailed csv files informing about a considered match (see [16] for more details). Still, in many cases, it remains difficult to decide if a match is right and we had to resort to a more time-consuming option: the exploration of the source code. In fact, those situations were not rare and the validation of our results took about 10 days of work.

\subsection{OO Design Differencing}

UMLDiff [31] is a "domain-specific structuraldifferencing algorithm" based on very detailed specifications of the diagrams they match. To the best of our knowledge, it remains the state of art algorithm on class diagram differencing and is probably one of the most cited differencing tools. It is available as an Eclipse plug-in (linked to a PostgreSQL database) and we used it (with the same parameters in [31]) for comparison with MADMatch.

\subsubsection{Objects}

Given that UMLDiff is coupled with a fact extractor that only works on Java programs, we chose four Java programs of various sizes: DNSJava, JFreeChart, ArgoUML, and Eclipse.

DNSJava ${ }^{9}$, the smallest program, is an open source Domain Name Server (DNS) written in Java. We selected the same 40 releases previously used by [2] in their paper about class evolution discontinuities. JFreeChart ${ }^{10}$ is a free Java chart library, studied in [31], which purpose is to help developers creating professional quality charts in their programs. ArgoUML ${ }^{11}$ is a medium-size, Java-based, UML development tool, which supports most of the standard UML diagrams and can also export data in a variety of formats, including XMI, C++, C\#, Java, and PHP source code. Eclipse $^{12}$ is a large, open-source, integrated development environment. It is mostly written in Java, with

\footnotetext{
9. http://www.dnsjava.org

10. http://www.jfree.org/jfreechart/

11. http:/ / argouml.tigris.org/

12. http://www.eclipse.org/
} 
$\mathrm{C} / \mathrm{C}++$ code used mainly for the widget toolkit. $\mathrm{C}++$ code is not considered in this study.

Class diagrams are modeled as reported in Table 1. We recover the class diagrams of the studied Java programs using the Ptidej tool suite ([13]), which includes a Java parser (for binaries) and a graph exporter dedicated to MADMatch.

Table 5 reports information about the diagrams extracted from the above programs: number of entities, relationships, etc.

\subsubsection{Accuracy of the Solutions}

Figure 6 presents descriptive statistics on the compared evaluation measures. For each program (DNSJava, JFreeChart, ArgoUML), we display boxplots for each technique (MADMatch and UMLDiff). There is no comparison data for Eclipse given that the size of Eclipse is intractable for UMLDiff (as confirmed by discussions with the authors of [31]).

From Figure 6, it is clear that (i) MADMatch and UMLDiff agree on large parts of their returned solutions and (ii) when they disagree MADMatch has higher differential recall and precision values.

The sets of matches returned by MADMatch contain the majority of the matches returned by UMLDiff: medians are of $100 \%$ for DNSJava, $94 \%$ for JFreeChart, and $90 \%$ for ArgoUML. In contrast, the intersection of UMLDiff and MADMatch accounts for a smaller subset of MADMatch solutions: medians are of $86 \%$ for DNSJava, 74\% for JFreeChart and 63\% for ArgoUML.

The differential precisions of MADMatch are higher than those of UMLDiff. For all three programs, it appears that the sets of matches exclusive to MADMatch are consistently and significantly more precise than those of UMLDiff. The medians of the differential precisions of MADMatch are $100 \%$ for DNSJava, $79 \%$ for JFreeChart, and 78\% for ArgoUML. They are substantially higher than those of UMLDiff: $42 \%$ for DNSJava, 67\% for JFreeChart, and 63\% for ArgoUML.

MADMatch also has higher differential recall values than UMLDiff. Most of the correct node matches brought by matches exclusive to one algorithm come from the sets of MADMatch with medians of $100 \%$, $82 \%$, and $74 \%$ respectively for DNSJava, JFreeChart, and ArgoUML. Detailed results obtained on each program are available in [16].

As for Eclipse, given that UMLDiff is unable to treat its diagrams, we do not have any compared accuracy measures to report. The number of nonperfect matches is quite high (1,733 from 1.0 to $2.0,827$ from 2.0 to 2.1, and 839 from 2.1 to 3.0). The Eclipse dataset was selected mainly to test the scalability of our approach, so we did not proceed to an extensive, costly manual validation of the results. We observed, though, precisions higher than 95 from a random sample $(2 \%)$ of the returned matchings.

Overall, the above results empirically validate some intrinsic advantages of MADMatch over UMLDiff.

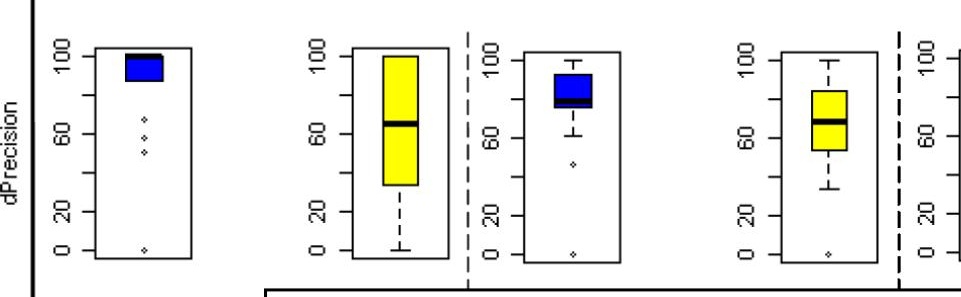

(b) MADMatch vs. UMLDiff: Differential Pr

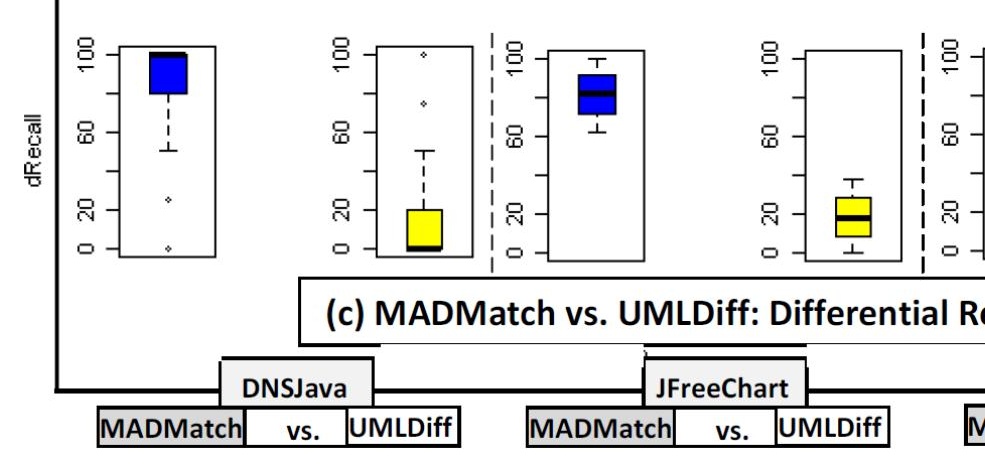

Figure 6. Boxplots of the compared accuracy measures from MADMatch versus UMLDiff

In particular, it obtains a much better recall, since unlike UMLDiff, it is able to recover merges and combinations of move and renaming (respectively operations 2 and 3 in the presentation of our running example). Another limitation of UMLDiff is that it builds a solution by adding node matches that, unlike MADMatch, cannot be reassessed later.

\subsubsection{Computation times}

We retrieve for MADMatch computation times when considering the different sizes of programs, from the smallest to the largest. Computation times of MADMatch observed for the different programs range from less than $1 \mathrm{sec}$ to about 9 hours. For DNSJava, MADMAtch takes from 0.1 to 30s with an average of 2.3s. Computation times are much higher for JFreeChart: from 2 to 1,390s with an average of 93s. The application of MADMatch on ArgoUML generates computation times ranging from 156 to 1,103s; the average being 537s.

On the same machine, UMLDiff took in total $18 \mathrm{~h} 40 \mathrm{~min}$ for JFreeChart. However, possibly due to its use of a backend database, the algorithm is highly sensitive to the computer load and the number of versions differenced at once ${ }^{13}$. Settings of the database may also affect computation times. In Xing's thesis ${ }^{14}$, the times reported for JFreeChart were of approximately $6 \mathrm{~h} 21 \mathrm{~min}$. Compared to the approximate 44 min MADMatch took, it is clear that UMLDiff is much slower. The same observation goes for DNSJava (40 min versus less than 2 min for MADMatch) and ArgoUML (about 8h30min versus 1h20min for MADMatch). As for Eclipse, MADMatch takes from

13. On average, we applied UMLDiff on five consecutive releases. 14. Z. Xing is the author of UMLDiff. 
Table 5

Summary of the object programs (MADMatch vs. UMLDiff)

\begin{tabular}{l|cr|c|c|c|c|}
\hline \multirow{2}{*}{ Programs } & \multicolumn{2}{|c|}{ Releases } & \multicolumn{4}{c|}{ Number of } \\
\cline { 2 - 7 } & \multicolumn{2}{|c|}{ Number Thereof) } & Entities & Relations & Classes & Methods \\
\hline DNSJava & $0.1-1.4 .3$ & $(40)$ & $607-1,765$ & $1,685-5,081$ & $39-105$ & $337-1,084$ \\
JFreeChart & $0.5 .6-1.0 .0$ & $(30)$ & $1,074-14,170$ & $2,722-41,792$ & $100-1,139$ & $714-9541$ \\
ArgoUML & $0.10 .1-0.26 .2$ & $(10)$ & $12,237-21,622$ & $27,415-59,676$ & $898-1,887$ & $7,402-14,895$ \\
Eclipse & $1.0-3.0$ & $(4)$ & $94,472-226,182$ & $317,471-746,466$ & $6,188-14,521$ & $58,948-141,811$ \\
\hline
\end{tabular}

$3 \mathrm{~h} 25 \mathrm{~min}$ (to match versions 1.0 and 2.0 ) to $9 \mathrm{~h}$ (for versions 2.1 and 3.0).

In conclusion, UMLDiff is much slower than MADMatch and is not scalable to Eclipse.

\subsection{API evolution}

We report in the following subsection a comparison of MADMatch with AURA [28], a state-of-the-art API evolution technique.

\subsubsection{Objects}

Regarding API evolution, we studied the same pairs of program versions used in the presentation of AURA [28]: JFreeChart 0.9.11 vs. 0.9.12, jEdit 4.1 vs. 4.2, JHotDraw 5.2 vs. 5.3 and Jakarta Struts 1.1 vs. 1.2.4. These are four medium-sized Java programs that include a text editor (jEdit), a chart library (JFreeChart), a framework for developing J2EE Web applications (Struts), and a Java GUI framework for technical and structured graphics (JHotDraw).

\subsubsection{Results}

Table 6 presents the comparison of MADMatch to AURA. We manually validated the matches contained in the intersection of both algorithms and found only one incorrect match in the total of 384 matches shared by the algorithms (on the four matchings reported in Table 6). This validation allows us to compute the precision of both algorithms on the considered case studies. When we sum up matches obtained for the four programs, MADMatch attains an overall precision of $89 \%$ (70 incorrect matches out of 613 ) while AURA stands at 84\% (90 incorrect matches out of 557). Correct matches exclusively found by MADMatch reach a total of 160 versus 84 for AURA. The differential precisions are of $70 \%(160 / 229)$ for MADMatch and 49\% (84/173) for AURA.

When we apply the differential measures for each program, we find that on average, MADMatch proposes a differential precision of $69 \%$ (vs. 33\% for AURA) and a differential recall of $74 \%$ (vs. $26 \%$ for MADMatch).

Most of the false positives returned by AURA come from its one-to-many and many-to-one correspondences. MADMatch includes a mechanism that is less tolerant to discrepancies involving multiple correspondences, and thus avoids in most cases the inclusion of big and inaccurate merges. Also, unlike
Table 7

Matching the specification to the Markov model

\begin{tabular}{l|l}
\hline Algorithm & Results \\
\hline All Agree on: & $(\{\mathrm{s} 0\},\{\mathrm{m} 0\}),(\{\mathrm{s} 1\},\{\mathrm{m} 3\}),(\{\mathrm{s} 2\},\{\mathrm{m} 5\})$, \\
& $\begin{array}{l}(\{\mathrm{s} 4\},\{\mathrm{m} 13\}),(\{\mathrm{s} 5\},\{\mathrm{m} 11\}),(\{\mathrm{s} 8\},\{\mathrm{m} 7\}), \\
(\{\mathrm{s} 9\},\{\mathrm{m} 15\}),(\{\mathrm{s} 13\},\{\mathrm{m} 14\}),(\{\mathrm{s} 15\},\{\mathrm{m} 16\})\end{array}$ \\
\hline PLTSDiff & $(\{\mathbf{s} 3\},\{\mathbf{m} 8\}),(\{\mathbf{s 1 2}\},\{\mathbf{m} 10\})$ \\
\hline MADMatch & $\left(\begin{array}{l}(\mathbf{s} 3\},\{\mathbf{m} 1, \mathbf{m} 8\}),(\{\mathbf{s} 6\},\{\mathbf{m} 2\}),(\{\mathbf{s} 7\},\{\mathbf{m} 4\}), \\
\end{array}\right.$ \\
\hline
\end{tabular}

AURA and many other API evolution approaches, MADMatch searches for a coherent matching, in which each correct match included in the solution provides a useful feedback for the identification of other correct node matches.

\subsection{Differencing Labeled Transition Systems}

We compared MADMatch with PLTSDiff [4] that is a recent algorithm proposed for the matching of LTS from a structural point of view.

\subsubsection{Modeling and objects}

In our modeling of LTS, states and transitions are represented as nodes that are linked using two types of arcs (from a state to a transition and vice-versa). In addition, when states are not labeled, we assign to them artificial names obtained from the concatenation of the labels of their surrounding transitions.

To verify the applicability of MADMatch on LTS, we used the exact same LTS used in PLTSDiff [4]. The matchings performed in [4] involved only three labeled transition systems: the specification of a small CVS client (S), the reverse-engineered Markov model (M) and the reverse-engineered EDSM model (E). Figure 6.4.1 displays the LTS $S$ and $M$.

\subsubsection{Results}

Table 7 presents the results of PLTSDiff and MADMatch when we match the specification $S$ to the Markov model M. PLTSDiff and MADMatch agree on most (9) matches. Divergences between both algorithms (bold faced) consist mostly of additional pairs of nodes - e.g., $(\{\mathrm{s} 6\},\{\mathrm{m} 2\}),(\{\mathrm{s} 7\},\{\mathrm{m} 4\})-$ in MADMatch. MADMatch includes the PLTSDiff results and extends them with other correct matches, such as $(\{\mathrm{s} 6\},\{\mathrm{m} 2\}),(\{\mathrm{s} 7\},\{\mathrm{m} 4\})$. The merge $(\{\mathrm{s} 3\}$, $\{\mathrm{m} 1, \mathrm{~m} 8\})$ - instead of $(\{\mathrm{s} 3\},\{\mathrm{m} 8\})$ for PLTSDiff reports accurately that the state $\mathrm{s} 3$ corresponds in fact (and this is obvious on Figure 6.4.1) to the states m1 


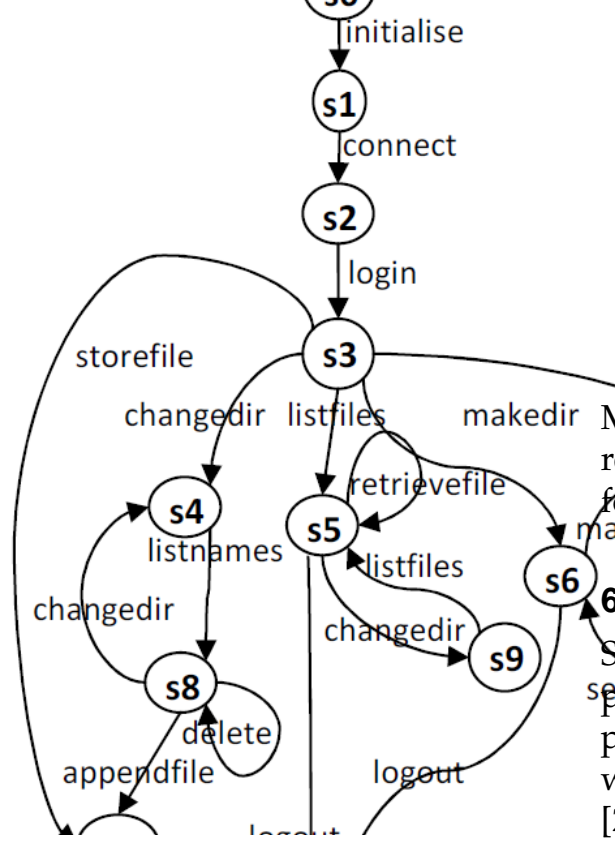

$=$ dRecall) set provides more insight because it can return acturate merges or splits that are out of reach for Pu syity (given its one-to-one limitation).

6.5 Dtokefile

6.5 Differèncingenguence Diagrams

Sequence S10 mrams (SD) model the behavior of a

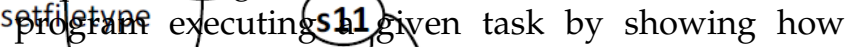
processep edodfollejects) perate with one another and in which order. Research work on matching SDs include [21], 2221, and an TBM tool in Rhapsody but we could not find engugh material (availability of tool, data, results etc.) for o comparison. Although sequences
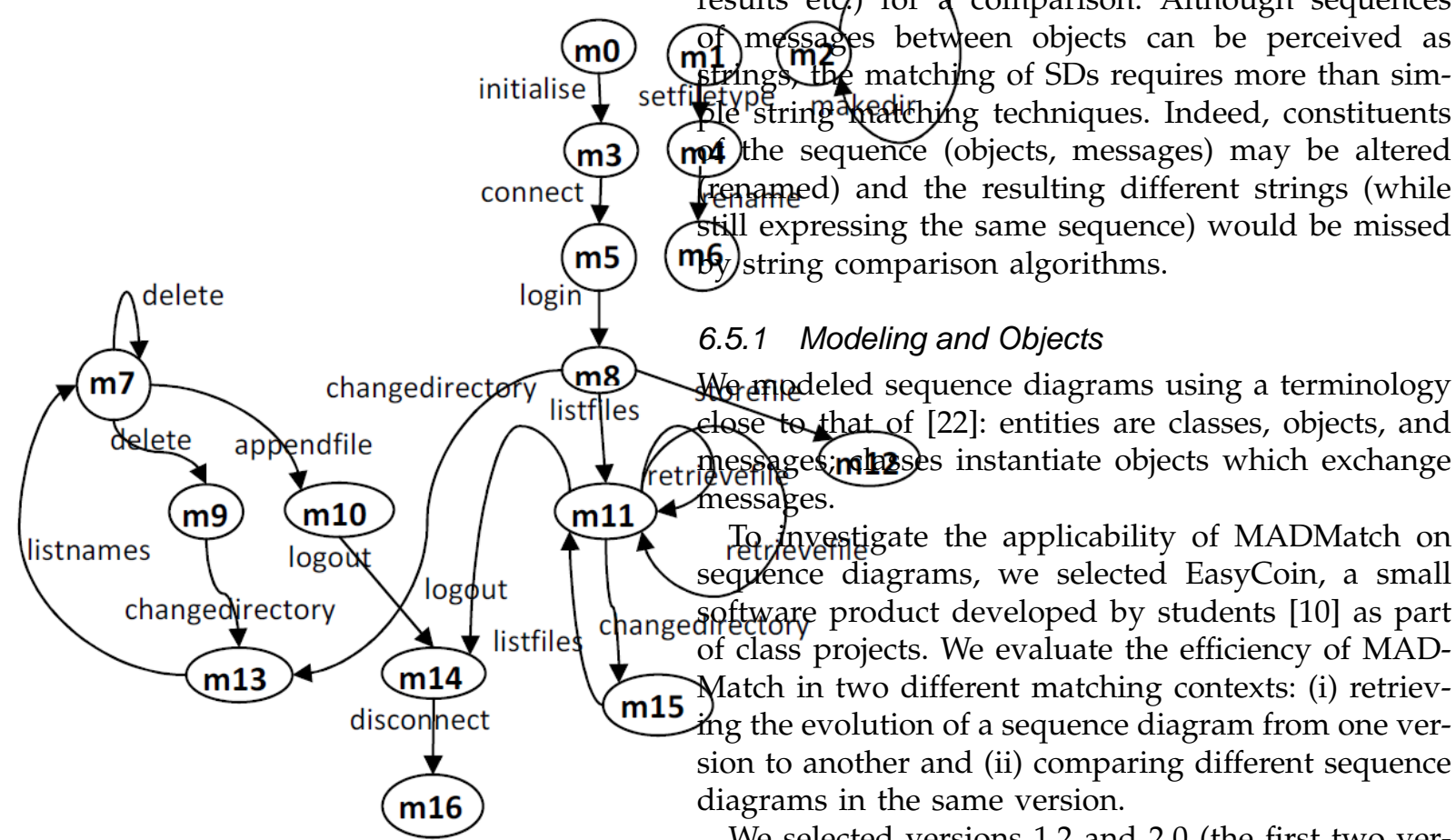

\subsubsection{Modeling and Objects}

(b) Labeled Transition System M

Figure 7. Labeled Transition Systems Used

and $\mathrm{m} 8$ of the Markov model. The additional match $(\{\mathrm{s} 12, \mathrm{~s} 14\},\{\mathrm{m} 10, \mathrm{~m} 12\})$ is hard to classify: states $\mathrm{m} 10$ and $\mathrm{m} 12$ (from the second LTS) can be seen as the result of the split of the state s12 (from the first LTS) but the merge of $s 14$ and $s 12$ is quite debatable ${ }^{15}$.

We observed similar results for the matching of the state machines $S$ and E. With respect to PLTSDiff,

15. In fact, less tolerant settings (dnr=dar=0.2) of MADMatch do not include such an operation.

Heforeded sequence diagrams using a terminology close to that of [22]: entities are classes, objects, and mesongeringes instantiate objects which exchange Tessages.

Teqryveftigate the applicability of MADMatch on sequence diagrams, we selected EasyCoin, a small softyare product developed by students [10] as part of class projects. We evaluate the efficiency of MADMatch in two different matching contexts: (i) retrieving the evolution of a sequence diagram from one version to another and (ii) comparing different sequence diagrams in the same version.

We selected versions 1.2 and 2.0 (the first two versions for which we have modeling data) of EasyCoin and three sequence diagrams out of those versions: InserireEnteEmettitore and ModificareEnteEmettitore (from version 1.2) and InserireEnteEmettitore (from version 2.0$)^{16}$

\subsubsection{Results}

Figure 8 presents the matching of the two subsequent versions of InserireEnteEmettitore. Except for a merge, all objects are perfectly matched. Messages propose a more interesting situation. In the figure, unmatched 


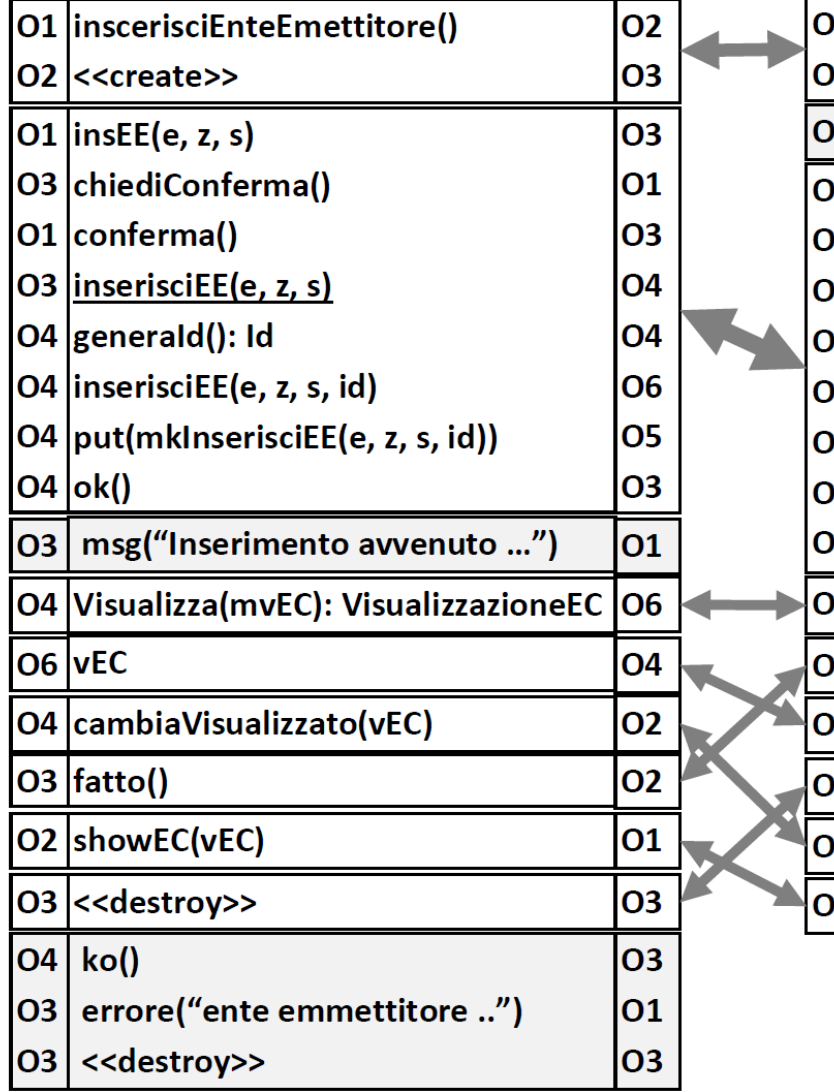

Figure 8. Matching InserireEnteEmettitore from version 1.2 to 2.0

messages are displayed on grey backgrounds while matched messages are linked by arrows. Additionally, we underlined non-perfectly matched messages (e.g., inserisciEE $(\mathrm{e}, \mathrm{z}, \mathrm{s})$ matched to inserisciEE(IE,e,z,s)). The figure reveals that the matching between the two subsequent versions of InserireEnteEmettitore consists in many contiguous matched segments. Some are long - e.g. insEE(e, z, s) to ok() - and some consist of only one message. We can also observe the re-ordering of some messages, which cause ruptures of segments that otherwise would be longer. This suggests that the order of the messages in those segments is not particularly important. An algorithm restricted to retrieving sequences of messages would most likely miss those matches. Overall, these results are $100 \%$ consistent with the oracle.

The same perfect alignment with the oracle is observed when applying MADMatch on InserireEnteEmettitore and ModificareEnteEmettitore. MADMatch reveals that both diagrams seem to have the same core behavior, with some few different specific operations. From discussions with authors of [10], we understood that students involved in the EasyCoin project usually copied/pasted then modified the diagrams. MADMatch is able to retrieve common patterns of behavior between sequence diagrams even when the messages actually matched - e.g., inserireEE() versus modificareEE()- are different. We believe that this is another advantage relatively to string matching techniques, which would be unable to match such

\subsection{Mining Evolution Information from JFreeChart}

In JFreeChart, information about the changes performed, from one version to the next, can be found in three different locations: the readme files, the change logs, and the source code files.

\subsubsection{The Readme files}

All JFreeChart versions, except the first one, contain a readme file with high level information on the changes performed since the last version. However, more often than not, the provided information is sparse, ambiguous and vague. We present below the content of the readme file of the second version (0.6.0).

0.6 .0 : (27-Nov-2001) New plots including scatter plot, stacked bar charts and $3 \mathrm{D}$ bar charts. Improved pie chart. Data interfaces and classes moved to the JCommon class library. New properties to control spacing on bar charts. New auto-tick mechanism. JFreeChartPanel now incorporates buffering, and popup menu. Javadocs revised. Fixed numerous bugs from version 0.5.6. Demo application updated.

\subsubsection{Change Logs}

Starting from version 0.9.17, JFreeChart source files include change logs, to which the readme files now refer "for more details". Information provided by the change logs is more precise, detailed and structured. Every piece of information is accompanied by a time stamp and a tag ([API], [NEW], or [BUG]). We display below excerpts from the first change log, the one from 0.9.16 to 0.9 .17 .

27-Jan-2004 : [BUG] Moved working line attribute from StandardXYItemRenderer to state object;...

03-Feb-2004 : [NEW] Modified the XYSeriesCollection class so that it implements the IntervalXYDataset interface...

25-Mar-2004 : [API] Moved Spacer class from org.jfree.chart to org.jfree.ui; ;... 


\subsubsection{Java files}

Source code files are the most helpful files for our mining of change information. All the java files of JFreeChart have a header that details the changes made to the class(es) they contain, from the renaming of methods to visibility changes made for attributes. In many cases, the headers even indicate, to some extent, changes concerning classes they are linked to. We display below a typical header found in JFreeChart java files.

***** ValueAxis.java $* * * * *$

* 18-Sep-2001 : Added standard header and fixed DOS encoding problem

* 23-Nov-2001 : Overhauled standard tick unit code (DG);

* 04-Dec-2001 : Changed constructors to protected, and tidied up default values (DG);

* 12-Dec-2001 : Fixed vertical gridlines bug (DG);

* 16-Jan-2002 : Added an optional crosshair, based on the implementation by Jonathan Nash (DG);

* 23-Jan-2002 : Moved the minimum and maximum values to here from NumberAxis, and changed the type from Number to double (DG);

The files' headers also sometimes provide clear and explicit information as to the genesis of a class, as illustrated below.

***** AbstractRenderer.java *****

* 22-Aug-2002 : Version 1, draws code out of AbstractXYItemRenderer to share with AbstractCategoryItemRenderer (DG);

and

***** HorizontalShapeRenderer.java *****

* 15-Jul-2002 : Version 1, based on LineAndShapeRenderer (DG);

For each version of JFreeChart, we systematically examined all new or modified files. Depending on the version, we had to analyse from half a dozen to hundreds of files. Such work required two weeks of validation of which results we put online ${ }^{17}$. For our systematic mining, we did not examine the source code beyond the headers. Such additional work would have required prohibitive time without any guarantee of a perfect recall.

\subsection{Analysis of Impacting Factors}

The factors affecting the performance of MADMatch are both internal and external.

Internal factors refer to various decisions made when conceiving MADMatch. For example, our reduction of the search space is based on a (contextual) similarity threshold (0.5) below which a node pair from the relevant graphs is filtered out. On JFreeChart versions, on average, such operation removes about $98 \%$ of all these node pairs but only $0.23 \%$ of the correct node matches. This quite effective reduction helps in guiding the search towards good and very narrow areas of the search space, which translate into near identical solutions even for non-deterministic versions of MADMatch. On average, when we consider 30 different runs for a given pair of JFreeChart versions, the intersection between all the returned solutions (the sets of non-perfect node matches) represents more than $98 \%$ of these solutions. Our (limited) investigation of MADMatch dynamics proved enough for

17. http://web.soccerlab.polymtl.ca/ sekpo/data/data.htm
Table 8

Our Metrics for External Impacting Factors

\begin{tabular}{l|l|l|l|l|l|l}
\hline & $G_{1}$ & $G_{2}$ & $G_{1 r}$ & $G_{2 r}$ & $X c_{1}$ & $X c_{2}$ \\
\hline Nodes & $n_{1}$ & $n_{2}$ & $n_{1 r}$ & $n_{2 r}$ & $\begin{array}{l}n_{1 x} \\
w n_{1 x}\end{array}$ & $\begin{array}{l}n_{2 x} \\
w n_{2 x}\end{array}$ \\
\hline Arcs & $m_{1}$ & $m_{2}$ & $m_{1 r}$ & $m_{2 r}$ & & \\
\hline \hline $\begin{array}{l}\text { Distinct } \\
\text { Terms }\end{array}$ & $n D T_{1}$ & $n D T_{2}$ & $n D T_{1 r}$ & $n D T_{2 r}$ & $\begin{array}{l}n D T_{1 x} \\
w D T_{1 x}\end{array}$ & $\begin{array}{l}n D T_{2 x} \\
w D T_{2 x}\end{array}$ \\
\hline $\begin{array}{l}\text { All } \\
\text { Terms }\end{array}$ & $n T_{1}$ & $n T_{2}$ & $n T_{1 r}$ & $n T_{2 r}$ & $\begin{array}{l}n T_{1 x} \\
w T_{1 x}\end{array}$ & $\begin{array}{l}n T_{2 x} \\
w T_{2 x}\end{array}$ \\
\hline
\end{tabular}

stable and competitive results. An in-depth study is reserved for future work.

The focus of the current section is on external factors affecting MADMatch's performance. These factors are based on the input graphs and their study provides insight on the level of difficulty of a matching task. We share with UMLDiff authors the intuitive belief that the "further" the versions considered, the more challenging the matching task. However, we wanted to assess this belief using definite lexical or structural metrics.

\subsubsection{Presentation of the impacting factors}

Our metrics are defined using three basic units of information (nodes, arcs, and lexical terms) and three different perspectives (the initial graphs, the relevant graphs, and the exclusive elements). More precisely, the numbers of nodes, arcs and terms are computed from the initial graphs $\left(G_{1}\right.$ and $\left.G_{2}\right)$ and the graphs relevant to the matching $\left(G_{1 r}\right.$ and $\left.G_{2 r}\right)$ as defined in Section 4. The third perspective is that of elements exclusive to one of the graphs (denoted by $X c_{1}$ and $X c_{2}$ ). We count the nodes without perfect correspondence from one graph to another and the terms present in only one of the graph. In addition, proportions of such exclusive elements are computed relatively to the graphs $G_{1 r}$ and $G_{2 r}$.

Table 8 summarizes the metrics described above. Note that, for terms, we considered both the overall number of terms ( $n T$ : the same term can be counted several terms) and the number of distinct terms $(n D T)$. Proportions of exclusive elements are represented by variables starting by the letter $w$; for example $w n_{1 x}=n_{1 x} / n_{1 r}$ is the proportion of nodes in $G_{1 r}$ that do not have a perfect match in $G_{2 r}$.

For each pair (metric $c_{1}$, metric $_{2}$ ), we also compute the difference $\left(\mid\right.$ metric $_{1}-$ metric $\left._{2} \mid\right)$ as well as the product (metric $c_{1} *$ metric $c_{2}$ ). Finally, we also considered as a distance metric the number of days between the two versions which diagrams are to be matched.

The computation of these metrics provides additional insight on the input graphs. For example, the range for the number of nodes exclusive to either graph is $[11,7433]$, down from $[64,9019]$ in the relevant graphs, and $[1074,14170]$ in the initial graphs. With respect to the vocabulary, the number of distinct terms grows from 243 in the initial release (0.5.6) to 1457 in the 1.0.0 release. This is 6 times the initial number but about twice weaker than the increase 
Table 9 MADMatch's accuracy for JFreeChart

\begin{tabular}{l|l|l|l|l|l}
\hline & Min & $Q_{1}$ & Median & $Q_{3}$ & Max \\
\hline precision & 50 & 82 & 90 & 96 & 100 \\
mrecall & 50 & 86 & 98 & 100 & 100 \\
arecall & 72 & 87 & 95 & 99 & 100 \\
af1 & 67 & 86 & 93 & 96 & 100 \\
\hline
\end{tabular}

in the number of nodes. The number of distinct terms exclusive to the first diagram (terms removed or modified from one diagram to the next) varies between 0 and 431, with an average of 53 . Conversely, the number of distinct terms exclusive to the second diagram varies between 3 and 467, with an average of 162 .

To assess the impact of structure and vocabulary on MADMatch performance, we computed correlation coefficients (Pearson and Spearman) between the metrics defined above and performance measures taken from MADMatch results. The Pearson coefficient measures the linear dependence between two variables while the Spearman coefficient is a rank-correlation measure. For Pearson (resp. Spearman) correlation, values between 0.3 and 0.5 (resp. between 0.4 and 0.7 ) are usually interpreted as indications of medium correlation while values above 0.5 (resp. above 0.7 ) indicate strong correlation.

\subsubsection{Accuracy evaluation}

For every pair of versions, we mined from the JFreeChart files a set $E$ of node matches accounting for the correspondences explicitly mentioned. With $M$ being the set of node matches returned by MADMatch, we consider $M-E$, its subset of node matches not explicitly mentioned in JFreeChart files. We then proceeded to the manual validation of these correspondences by examining (when in doubt) lines of code from the java files. From this validation, we obtain a set $I$ of implicitly correct node matches that contains validated elements of $M$ as well as other correct node matches found while examining the source code.

Using the above defined sets, we computed the following accuracy measures:

- the percentage of correct node matches in MADMatch results: precision $=|M \cap(E \cup I)| /|M|$;

- the percentage of explicitly correct node matches retrieved by MADMatch: mrecall $=|M \cap E| /|E|$;

- the percentage of the correct node matches retrieved by MADMatch: arecall $=|M \cap(E \cup I)| / \mid E \cup$ $I \mid ;$

- an approximate measure of the overall accuracy: $a f_{1}=2 *($ precision $*$ arecall $) /($ precision + arecall $)$

Table 9 presents the five-number summary of these accuracy measures on JFreeChart. With the exception of some pairs of versions, results are generally excellent and all medians are above $90 \%$. On average, the manual validation of MADMatch results recovers $17 \%$ more correct node matches (with respect to the mined changes); almost all (93\%) these correct matches come from MADMatch results.

The relation between accuracy values and our impacting factors is summarized in Table 10, which reports the highest correlation values ${ }^{18}$ per metric category; weak correlation values are omitted and represented by ".".

There are a number of observations to be made based on these numbers:

- Metrics based on distinct terms always have better correlations with accuracy measures than the ones based on all the terms. In other words, the size of the lexicon used in the diagrams is more important than the number of times the terms of this lexicon are used.

- Except for mrecall, metrics based on the distinct terms always present higher correlation values than all the other categories of metrics. In particular, distinct terms found exclusively in either graph (but especially the first) are strongly correlated (up to -0.81) with the precision and the general accuracy measure $\left(a f_{1}\right)$. It would seem that the real challenge comes from the modification of the existing lexicon, not really from its extension.

- At best, our metrics have medium correlation with either of the two recall measures.

- The number of days between versions has no linear correlation with the accuracy measures but does present moderate rank correlation with the recall measures.

\subsubsection{Computation time evaluation}

The 5-number summary of the computation times on JFreeChart is (Min, $Q_{1}$, Median, $Q_{3}$, Max $)=(1,5$, 11, 32, 1390). MADMatch returns a solution within $1 \mathrm{sec}$ from version 0.7.2 to 0.7.3, with $n_{1 r}=67$ and $n_{2 r}=76$. On the other hand, from version 0.9.7 to 0.9.8, the computation takes up to 1390 seconds, with $n_{1 r}=7340$ and $n_{2 r}=7438$.

Table 11 summarizes the correlation coefficients of the impacting factors with the computation time. We make the following observations:

- The strongest correlation (0.98) is positive and obtained when we consider the product of the number of nodes exclusive to either graph $\left(n_{1 x}{ }^{*} n_{2 x}\right)$. This is unsurprising because this metric actually represent the number of pairs for which we do not know a priori whether they should be part (or not) of the sought matching.

- For the computation time, the number of terms (including redundant ones) is more important than the number of distinct terms. Strong positive correlation is observed with the product of the numbers extracted from both graphs (e.g.,

18. All p-values are below 0.05 . 
Table 10

Correlation between Impacting Factors and Accuracy Measures ( $\mathrm{P}=$ Pearson, $\mathrm{S}=$ Spearman)

\begin{tabular}{l|l|l|l|l|l}
\hline & & Nodes & Arcs & Terms & NbDays \\
\hline precision & $\mathrm{P}$ & $\left|n_{2 r}-n_{1 r}\right|:-0.46$ & $\left|m_{2 r}-m_{1 r}\right|:-0.44$ & $w D T_{1 x}:-0.81$ & 0 \\
& $\mathrm{~S}$ & $\left|n_{2 r}-n_{1 r}\right|:-0.46$ & $\left|m_{2 r}-m_{1 r}\right|:-0.47$ & $w D T_{1 x} * w D T_{2 x}:-0.69$ & -0.27 \\
\hline mrecall & $\mathrm{P}$ & $n_{2 r}:-0.42$ & $m_{2}:-0.31$ & $n D T 2:-0.37$ & $\approx 0$ \\
& $\mathrm{~S}$ & $n_{2 r}:-0.42$ & $m_{2 r}:-0.55$ & $n D T_{2 r}:-0.45$ & -0.56 \\
\hline arecall & $\mathrm{P}$ & - & - & $n D T_{2 x}:-0.38$ & 0 \\
& $\mathrm{~S}$ & - & - & $n D T_{2 x}:-0.54$ & -0.48 \\
\hline af1 & $\mathrm{P}$ & $\left|n_{2 r}-n_{1 r}\right|:-0.48$ & $\left|m_{2 r}-m_{1 r}\right|:-0.44$ & $w D T_{1 x}:-0.63$ & 0 \\
& $\mathrm{~S}$ & $\left|n_{2 r}-n_{1 r}\right|:-0.51$ & $\left|m_{2 r}-m_{1 r}\right|:-0.51$ & $\left|n D T_{2 x}-n D T_{1 x}\right|:-0.62$ & -0.31 \\
\hline
\end{tabular}

Table 11

Correlation between Impacting Factors and computation time ( $\mathrm{P}=$ Pearson, $\mathrm{S}=$ Spearman)

\begin{tabular}{l|l|l|l|l}
\hline & Nodes & Arcs & Terms & NbDays \\
\hline $\mathrm{P}$ & $n_{1 x}{ }^{*} n_{2 x}: 0.98$ & $m_{2 r}-m_{1 r}: 0.81$ & $\begin{array}{l}n T_{1 r}{ }^{*} n T_{2 r}: 0.86 \\
w T_{2 x}:-0.68\end{array}$ & 0.33 \\
\hline $\mathrm{S}$ & $n_{1 x}{ }^{*} n_{2 x}: 0.87$ & $m_{2 r}-m_{1 r}: 0.93$ & $\begin{array}{l}n T_{1 r}{ }^{*} n T_{2 r}: 0.90 \\
w T_{2 x}:-0.57\end{array}$ & 0.63 \\
\hline
\end{tabular}

Pearson $\left(n T_{1 r}{ }^{*} n T_{2 r}\right.$, time $\left.)=0.86\right)$. Strong negative correlation is also observed, notably with the proportion of terms exclusive to the second graph (Pearson $\left(w T_{2 x}\right.$, time $\left.)=-0.68\right)$. This may be because the dissemination of a lot of new terms is more likely to lead to a greater and quicker reduction of the search space by providing a more clear contrast between the termal contexts of nodes taken from both graphs.

- The number of days separating the versions yields only a moderate correlation with the computation time.

\subsection{Of Changes and Challenges}

The solutions returned by MADMatch miss some correct node matches and return some incorrect ones. In our case study, many of these incorrect matches involve demo and test classes with similar names and weak connectivity. These classes also often share many generic methods such as suite(), testSerialisation(), testEqual(), main() etc. and are thus particularly challenging for MADMatch (and UMLDiff as well). In general, incorrect matches can be easily detected thanks to our cost mechanism. In a solution returned by MADMatch, each node match is assigned a value (vnm) accounting for its contribution to lowering the overall cost. Node matches which contributions are below the average $\left(s \_a v g\right)$ in the overall solution tend to be incorrect. On average, node matches that satisfy $v n m \leq s \_a v g / 4$ are $69 \%$ incorrect, represent $5 \%$ of a solution but account for $60 \%$ of all the incorrect matches. Similarly, node matches that satisfy $v n m \leq s_{-} a v g / 2$ are, on average, $43 \%$ incorrect, represent $11 \%$ of a solution but account for $83 \%$ of all the incorrect matches. The current section is devoted to the investigation of the challenges posed by the different types of changes we observed in JFreeChart.

\subsubsection{Basic Changes}

In a class diagram, when an entity is matched to another, there are five main ways in which they can differ: (i) their name, (ii) the name of the entities that contain them, (iii) their modifier (e.g., visibility), (iv) their input (if they are methods), and (v) their type or return type (if they are methods or fields). The first two possibilities refer to a renaming and the last three can be regrouped as changes in the features.

7.3.1.1 Entity Renaming: The first two basic changes refer to a renaming, which is performed by reordering, adding, deleting, correcting, or replacing the terms composing an entity name. Such operations are relatively frequent in JFreeChart and this suggests a high interest for vocabulary accuracy and clarity. It may be that, as a class library used by other developers, JFreeChart has high standards for vocabulary intuitiveness and naming conventions. Consequently, it is not rare to observe many rounds of renaming on an entity to reflect accurately its role. For example, the class Swing in version 0.5.6 has been renamed three times: SwingRefinery (in 0.6.0), JRefineryUtilities (in 0.7.0), and RefineryUtilities (in 0.9.0). The last renaming reflects a shift of this "collection of utility methods relating to user interfaces" from the javax.swing library (with its cohort of $J^{*}$ classes) to the java.awt library. We provide in Table 12 a tentative classification of renaming purposes. Examples displayed in this table show the range and complexity of the renamings. These alterations strongly suggest that, for effective matching, text similarity measures, however advanced, need to be used in conjunction with structural information.

7.3.1.2 Feature changes: For access modifiers (private, public, protected), we observed all possible transformations and some changes were applied in such a large scale that they were mentioned in readme files: e.g., "most 'protected' member variables have been changed to 'private'" in 0.9.4. As for changes of type, aside from JFreeChart classes, they also involve java library classes (String, Class, Object, Point2D, Window) and primitives (int, double, float, etc.). These low-level changes are interesting because they can indicate extensions (boolean $\rightarrow$ int $\rightarrow$ double), simplifications (Integer $\rightarrow$ int), clarifications (double [] $\rightarrow$ Point2D) or library changes (javax.swing. ${ }^{*} \rightarrow$ java.awt. ${ }^{*}$ ). Also interesting are cases in which a java library class is replaced by a 
Table 12

Tentative classification of Renaming Purposes

\begin{tabular}{l|l|l}
\hline Purpose & Simple (one-term) & Complex (2+ terms) \\
\hline Clarify? & $\begin{array}{l}\mathrm{t} \rightarrow \text { transform } \\
\text { STANDARD } \rightarrow \text { FORWARD } \\
\text { dialType } \rightarrow \text { shape } \\
\text { type } \rightarrow \text { extent (context: range) } \\
\text { pair } \rightarrow \text { item }\end{array}$ & $\begin{array}{l}\text { b2s } \rightarrow \text { boundToString } \\
\text { getStart } \rightarrow \text { getFirstMilliSecond } \\
\text { drawArcFor } \rightarrow \text { drawArcForInterval }\end{array}$ \\
& $\begin{array}{l}\text { JFreeChartDemo2 } \rightarrow \text { TimeSeriesDemo5 } \\
\text { limitPieDataset } \rightarrow \text { createConsolidatedPieDataset }\end{array}$ \\
\hline Simplify? & ACTION_COMMAND $\rightarrow$ COMMAND & $\begin{array}{l}\text { recalculateIntervalWidth } \rightarrow \text { recalculateInterval } \\
\text { addEntities } \rightarrow \text { addAll, addEntity } \rightarrow \text { add (EntityCollection) }\end{array}$ \\
\hline Specify? & listeners $\rightarrow$ listenerList & getOutliers $\rightarrow$ getOutliersArray \\
\hline Extend? & vertical $\rightarrow$ angle & DataSourceNotCompatibleException $\rightarrow$ PlotException \\
\hline Correct? & Icone $\rightarrow$ Icon & $\begin{array}{l}\text { FIRST_MINUTE_IN_DAY } \rightarrow \text { FIRST_MINUTE_IN_HOUR } \\
\text { Axisocation } \rightarrow \text { AxisLocation }\end{array}$ \\
\hline$? ? ?$ & LEFT $\rightarrow$ RIGHT & dayOfWeek $\rightarrow$ weekday \\
\hline
\end{tabular}

new JFreeChart class (e.g., org.apache.log4j.Logger $\rightarrow$ org.jfree.util.LogContext).

7.3.1.3 MADMatch performance: Challenging situations occur when many basic changes are applied at once. Starting from 3 basic changes on the same entity, the precision of MADMatch falls below 50\% (at 39\%) but the recall remains high (81\%). For entity matches with 4 or more basic changes, the precision further drops at around $20 \%$ but the recall remains steady $(78 \%)$. Overall, the higher the number of basic changes in a node match, the likelier it is an error. On the other side, our experiments suggest that node matches with a high number of changes are not necessarily harder to retrieve, at least for MADMatch. We found in all JFreeChart evolution 232 cases with 3 changes, 38 cases with 4 changes and 4 cases with 5 changes. Table 13 provides an example for each case.

\subsubsection{Switches}

A switch is a pair of node matches of the form $(X, Y)$ and $(Y, X)$ in which two entities $X$ and $Y$ keep the same identity (name and features) from one version to another but swap their "body". Such cases run counter to the assumption made by UMLDiff according to which entities sharing the same identity should be paired in a definitive way. In JFreeChart, we encountered several cases of switches as evidenced by the headers' excerpts below.

***** CategoryDataset.java ***** $(0.9 .8)$

* 23-Apr-2003 : Switched CategoryDataset and KeyedValues2DDataset so that CategoryDataset is the super interface

***** CombinedDataset.java ***** $(1.0 .0)$

* 15-Jul-2004 : Switched getX() with getXValue() and getY() with getYValue() (DG);

From version 0.9 .20 to 1.0.0, there are tens of cases in which the methods $\operatorname{get}^{*}()$ and get*Value() in a given class are switched. MADMatch was able to recover most $(78 \%)$ of these switches because it allows the reconsideration of perfect node matches if they do not have perfectly matched neighborhoods. Often, when two given entities are switched, their respective neighborhoods (especially the entities that use them) tend to change as well.

\subsubsection{Merges}

When we only consider merges, MADMatch has, on average, a precision of $68 \%$ and a recall of $52 \%$ but these numbers do not tell the whole story. Table 14 presents some examples of merges returned by MADMatch, whether accurately (true positives) or not (false positives) as well as merges that are missed (the true negatives). We can observe that MADMatch is able to retrieve a large variety of mergesplit operations. Inaccurate merges result mostly from the erroneous addition of elements that should have been unmatched. Interestingly, some switches confuse MADMatch, which instead of actual switches $\left(X_{1}, Y_{2}\right)$ and $\left(Y_{1}, X_{2}\right)$ prefers to return many-to-many matches $\left(\left\{X_{1}, Y_{1}\right\},\left\{X_{2}, Y_{2}\right\}\right)$.

The failure to retrieve some accurate merges occurs mostly when clones are involved. Clone detection is beyond the scope of MADMatch but we considered cases where explicit information about the origin of a given entity is given (e.g., "Version 1, based on ..."). In most of these cases, MADMatch fails and this may well be by design. Indeed, if between two versions, an entity $X$ is not submitted to any change but is copied and renamed $Y$, MADMatch may consider the match $(X, X)$ as definitive and will not try to recover $(\{X\} \rightarrow$ $\{X, Y\})$. As a result, many clones are lost. When clones are ignored, the average recall increases dramatically from 52 to $84 \%$.

In some other cases, MADMatch fails to return all the nodes involved in a merge when these nodes exceed three or four. Large numbers of merges and splits occurring between two versions also present serious challenges for MADMatch because they collectively result in a significant and complex alteration. For instance, between versions 0.9.8 and 0.9.9, MADMatch failed to recover completely 242 merges because tens of classes (and their methods and fields) had been cloned, merged or split.

\subsubsection{Moves}

Moving an entity from a package/class to another is relatively common in JFreeChart. In some cases, the move is made towards a more specialised class: e.g., the method rotateShape is moved from RefineryUtilities 
Table 13

Examples of node matches with 3 or more basic changes

\begin{tabular}{|c|c|c|c|c|c|c|}
\hline$\#$ & modifier & type & parent & name & input & \\
\hline 3 & & & $\begin{array}{l}\text { JFreeChart } \\
\text { ChartFactory }\end{array}$ & $\begin{array}{l}\text { createHiLowChart } \\
\text { createHighLowChart }\end{array}$ & $\begin{array}{l}\text { XYDataSource } \\
\text { String, String, String, HighLowDataset, boolean }\end{array}$ & $\begin{array}{l}0.5 .6 \\
0.6 .0\end{array}$ \\
\hline 4 & $\begin{array}{l}\text { protected } \\
\text { public static }\end{array}$ & & $\begin{array}{l}\text { Axis } \\
\text { RefineryUtilities }\end{array}$ & $\begin{array}{l}\text { drawVerticalString } \\
\text { createHighLowChart }\end{array}$ & $\begin{array}{l}\text { String, Graphics2D, float, float } \\
\text { String, Graphics2D, float, float, double }\end{array}$ & $\begin{array}{l}0.8 .0 \\
0.9 .0\end{array}$ \\
\hline 5 & $\begin{array}{l}\text { public } \\
\text { public static }\end{array}$ & CategoryDataset & $\begin{array}{l}\text { DefaultCategoryDataset } \\
\text { DatasetUtilities }\end{array}$ & $\begin{array}{l}\text { DefaultCategoryDataset } \\
\text { createCategoryDataset }\end{array}$ & $\begin{array}{l}\text { double[][] } \\
\text { String, String, double[][] }\end{array}$ & $\begin{array}{l}0.9 .4 \\
0.9 .5\end{array}$ \\
\hline
\end{tabular}

Table 14

Some examples of Merges

\begin{tabular}{|c|c|c|c|}
\hline & Operation & Example & version \\
\hline \multirow{7}{*}{ True Positive } & Split Class & $\begin{array}{l}\text { JFreeChart } \rightarrow \text { JFreeChart and JFreeChartConstants } \\
\text { AxisLocation } \rightarrow \text { AxisLocation and RectangleEdge }\end{array}$ & $\begin{array}{l}0.7 .4 \\
0.9 .10\end{array}$ \\
\hline & Merge Class & $\begin{array}{l}\text { BarPlot and CategoryPlot } \rightarrow \text { CategoryPlot } \\
\text { JFreeChart and JFreeChartConstants } \rightarrow \text { JFreeChart }\end{array}$ & $\begin{array}{l}0.8 .0 \\
0.9 .19\end{array}$ \\
\hline & Split and rename & [StandardCategory]ItemLabelGenerator $\rightarrow$ LabelGenerator and ToolTipGenerator & 0.9 .19 \\
\hline & Split a constructor & ChartTiming4 ()$\rightarrow$ ChartTiming4() and run( ) & 0.9 .9 \\
\hline & Keep Deprecated & getMaximumAxisValue $\rightarrow$ getMaximumAxisValue and getUpperBound & 0.9 .12 \\
\hline & Make a back-up & JFreeChartInfo $\rightarrow$ JFreeChartInfo and JFreeChartInfoOld & 0.9 .2 \\
\hline & Perfect Split & drawOutlineAndBackground $\rightarrow$ drawOutline and drawBackground & 0.9 .5 \\
\hline \multirow{3}{*}{$\begin{array}{l}\text { False Positive } \\
\text { (confusion with) }\end{array}$} & deleted element & [SystemProperties] Table, TableModel $\rightarrow$ TableModel & 0.6 .0 \\
\hline & new element & [Sortable] TableHeaderListener $\rightarrow$ Table, TableHeaderListener & 0.6 .0 \\
\hline & Switch & CategoryDataset, KeyedValues2DDataset $\rightarrow$ CategoryDataset, KeyedValues2DDataset & 0.9 .8 \\
\hline \multirow{2}{*}{ False Negative } & Clones & $\begin{array}{l}\text { LineAndShapeRenderer } \rightarrow \text { LineAndShapeRenderer, HorizontalShapeRenderer } \\
\text { GanttDemo } \rightarrow \text { GanttDemo1, GanttDemo2 }\end{array}$ & $\begin{array}{l}0.9 .3 \\
0.9 .5\end{array}$ \\
\hline & High Cardinality & $\begin{array}{l}\text { MADMatch: chart } \rightarrow \text { chart, chart.axis, chart.renderer } \\
\text { Oracle: chart } \rightarrow \text { chart, chart.axis, chart.renderer, chart.plot }\end{array}$ & $\begin{array}{l}0.9 .5 \\
0.9 .5\end{array}$ \\
\hline
\end{tabular}

to ShapeUtils. In others, the inverse is true: e.g., the method createDataset is moved (and renamed createSampleGanttDataset) from GanttDemo to DemoDatasetFactory. Due to scalability issues, UMLDiff cannot handle the move and renaming of a given entity and misses, as a result, many correct matches returned by MADMatch.

\subsection{Perspectives: Refactoring Decisions}

From one version to another, changes on the diagram entities can be counted by hundreds but most of these can often be explained by a much more reduced set of high-impact changes.

\subsubsection{Changes and Hierarchy}

Changes occurring on higher level entities affect lower-level entities. For instance, when the root of an application is changed as observed in JFreeChart (from com.jrefinery in version 0.9.7 to org.jfree in version 0.9 .8 ), the impact is observable on every node match. Similar but less important impact is also observed for package restructuring. For instance, from 0.5.6 to 0.6.0, all layout classes contained in the package com.jrefinery.util.ui of JFreeChart were regrouped in a new package com.jrefinery.layout.

\subsubsection{Renaming Impact}

Often, the node matches recovered by MADMatch reflect important renaming decisions. For example, from JFreeChart 0.5.6 to 0.6.0, DataSource is replaced by Dataset in many class names (e.g., chart.DataSource to data.Dataset or chart.DataSources to data.Datasets).
Table 16

\section{Some examples of Renaming Decisions}

\begin{tabular}{l|l}
\hline Renaming & Versions \\
\hline color $\rightarrow$ paint & $0.7 .2,0.9 .3,0.9 .12$ \\
colour $\rightarrow$ paint & $0.9 .4,0.9 .12$ \\
\hline data $\rightarrow$ dataset & $0.9 .0,0.9 .10,1.0 .0$ \\
${ }^{*}$ Utils $\rightarrow{ }^{*}$ Utilities [for Dataset, File, ...] & $0.7 .4,1.0 .0$ \\
\hline${ }^{*}$ List $\rightarrow{ }^{*}$ s (e.g., propertyList $\rightarrow$ properties) & 0.7 .4 \\
${ }^{*} \mathrm{~s} \rightarrow{ }^{*}$ List (e.g., listeners $\rightarrow$ listenerList) & $0.9 .3,0.9 .4$ \\
\hline${ }^{*}$ StackedHorizontal ${ }^{*} \rightarrow{ }^{*}$ HorizontalStacked ${ }^{*}$ & $0.9 .0,0.9 .1$ \\
\hline
\end{tabular}

Sometimes, the renaming in itself carries meaning about which changes were performed. For instance, from JFreeChart 0.9.1 to 0.9.2, many methods went from display $X()$ to create $X()$ (with $X$ being PieChartOne, etc.). Source code inspection reveals that in 0.9.1, display $X()$ functions were used to both create and display objects $X$ while in 0.9 .2 , the display tasks are aggregated and delegated to another method. Table 16 presents some recurrent, high impact, and relatively simple renaming over several versions.

Sometimes, the renaming decisions require elaborate regular expressions. For instance, from JFreeChart 0.9.16 to 0.9.17, the renamings (checkWindNull, WindNullCheckBox) and (checkShipNull, ShipNullCheckBox) may be summarized by the rule (checkXNull,

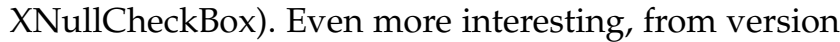
0.9.20 to 1.0.0, the renaming rule XPropertyEditPanel $\rightarrow$ DefaultXEditor (with $\mathrm{X}=$ Axis, Chart, Title, Plot, NumberAxis, ColorBar) summarizes tens of entity (classes, fields and methods) renaming cases. 
Table 15

Some examples of Moves

\begin{tabular}{l|l|l}
\hline Operation & Example & Version \\
\hline Move & rotateShape of RefineryUtilities moved to ShapeUtils & 0.9 .17 \\
& calculateRotatedStringBounds of RefineryUtilities moved to TextUtilities & 1.0 .0 \\
\hline Move + & JFreeChart.data $\rightarrow$ Plot.dataset & 0.9 .0 \\
Renaming & GanttDemo.createDataset $\rightarrow$ DemoDatasetFactory.createSampleGanttDataset & 0.9 .4 \\
& PiePlot.drawMultiplePies $\rightarrow$ MultiplePiePlot.draw & 0.9 .17 \\
& chart.CrosshairInfo $\rightarrow$ chart.plot.CrosshairState & 0.9 .17 \\
\hline
\end{tabular}

\subsubsection{Retrieving and enforcing refactoring decisions}

Hierarchy and term replacement impact are only one aspect of the underlying refactoring rules that we discovered while analysing JFreeChart evolution. For example, from version 0.5.6 to 0.6.0, a tentative rule expressed as follows: "the first parameter is removed if its type is JFreeChart" is enough to explain 15 node matches but is inconsistent with a couple of correct node matches. In most cases, such inconsistencies are the result of incomplete rules; in the example, the rule should have been restricted to methods of Plot classes. However, in many cases, the inconsistencies highlight hidden problems. For instance, from JFreeChart 0.9.4 to 0.9.5, the methods addTitle in the classes JFreeChart and JThermometerPlot were renamed respectively addSubTitle and addSubitle. The typo (Subitle) went unnoticed till the version 0.9.20 and was only corrected by version 1.0.0.

We identified two interesting phenomenon we call refactoring decision (RD) lag or reversal.

We observe an RD lag when a refactoring decision initiated on a given release is completely enforced only many releases later. For example, from JFreeChart 0.9.8 to 0.9.9, many pairs of classes (HorizontalX, VerticalX) were merged into a new class $X$. The same type of merges were still being performed in 0.9.10 and 0.9.12 for classes already present in 0.9.8.

Equally interesting are cases of RD reversals, which we define as situations in which a developer reverse a refactoring decision previously made. For example, the renaming rule "XList $\rightarrow X s$ " initiated in version 0.7.4 got reversed in versions 0.9.3 and 0.9.4. In some cases, these reversals are explicitly mentioned in the Java files' headers.

*****TimeSeries.java ${ }^{* * * * *}$

07-Dec-2001 : Renamed TimeSeries -> BasicTimeSeries (DG);

28-Jan-2003 : Changed name back to TimeSeries (DG);

*****JFreeChart.java*****

06-Mar-2002 : Moved constants to JFreeChartConstants interface (DG);

27-May-2004 : Moved constants from JFreeChartConstants.java back to this class (DG);

Starting from the version 0.9.4, JFreeChart authors started using CheckStyle, to help in their enforcement of good programming styles. We believe that JFreeChart would have also benefited from a tool able to collect refactoring decisions and highlight their inconsistencies or incomplete enforcement. For example, when a programmer decides to replace a term (e.g., data to dataset in 0.9.0), this should be stored and highlighted in relevant situations so that other developers avoid the re-introduction or preservation of the replaced term. By version 1.0.0 (20+ releases and 3 years and half later), JFreeChart developers were still "busy" renaming data into dataset. Such delays can have direct and negative impact. For example, from version 0.9.9 to 0.9.10, 13 methods refreshTicks*() of Axis classes underwent some change in their input parameters. All 13 changed the type of the last parameter (AxisLocation to RectangleEdge) but only 8 methods inserted a new parameter. It turns out that the other 5 methods should have also inserted that new parameter and the failure to do so generated a bug that was fixed in version 0.9.12.

\section{Conclusion}

Diagrams are very common representations of programs in software engineering. Whether conceived or retrieved from actual implementations, they convey important knowledge and abstract the programs to which they are related. Matching diagrams is an important task in many activities but it has mainly been researched only within a given scenario and on a given artifact.

MADMatch is a fast and scalable approach that addresses diagram matching as a graph matching problem, which is solved using tabu search. It includes new concepts that allow the handling of manyto-many matching in an efficient way. In this paper, we primarily and extensively evaluated MADMatch on class diagrams but we also reported experiments on sequence diagrams and labeled transition systems, which show that the approach is applicable to different kinds of diagrams. The compared evaluation of MADMatch with recent dedicated algorithms showed that our approach is more accurate and scalable than these previous approaches. We discussed extensively our results and tried to convey some of the insights gained from our application of MADMatch for the differencing of class diagrams.

Future work includes the application of MADMatch on other kinds of diagrams (log graphs, build dependency graphs) as well as the investigation of new insights for software engineering. In particular, from a software evolution perspective, we plan to use MADMatch for the study of vocabulary evolution in software diagrams. We are also very interested in analysing the consistency of changes occurring between two versions. Our case studies suggest that de- 
velopers could benefit from recommendations based on the identification and analysis of the refactoring decisions that explain the changes between two subsequent diagrams.

\section{APPENDIX: RUNNING EXAMPLE}

In this appendix, we described step-by-step application of MADMatch on the running example, with the costs $(d n r, d a r, a w$, asy, $n w)=(0.7,0.7,0.2,1,0.5)$.

\subsection{Retrieve basic costs}

Given the aggregate parameters, MADMatch uses an arbitrary base value (baseValue) from which it recomputes all the basic costs. To ease the reading, we will use baseValue $=100$.

- $C_{n+}=$ baseValue $=100$

- $a s y=1 \Rightarrow C_{n-}^{1}=C_{n-}^{2}, C_{a+}^{1}=C_{a+}^{2}, C_{a-}^{1}=C_{a-}^{2}$

- $d n r=0.7 \Rightarrow C_{n-}^{1}+C_{n-}^{2}=C_{n+} * 0.7=70$

- $a w=0.2 \Rightarrow C_{a+}^{1}+C_{a+}^{2}=C_{n+} * 0.2=20$

- $d a r=0.7 \Rightarrow C_{a-}^{1}+C_{a-}^{2}=\left(C_{a+}^{1}+C_{a+}^{2}\right) * 0.7=14$

We obtain $\left(C_{n+}, C_{n-}^{1}, C_{n-}^{2}, C_{a+}^{1}, C_{a+}^{2}, C_{a-}^{1}, C_{a-}^{2}, n w\right.$, $f w)=(100,35,35,10,10,7,7,0.5,0.5)$.

\subsection{Initialisation}

The first graph contains 11 nodes and 21 arcs while the second is made of 12 nodes and 19 arcs. An empty solution, with no matches, thus costs $\left(11 C_{n-}^{1}+21\right.$ $\left.C_{a-}^{1}\right)+\left(12 C_{n-}^{2}+19 C_{a-}^{2}\right)=1085$. MADMatch initializes the solution $S$ with the perfect matches (\{root\}, $\{$ root $\}),(\{$ Ticket $\},\{$ Ticket $\}),(\{$ Lottery $\},\{$ Lottery $\})$, and (\{restart $\},\{$ restart $\})$ that generate savings of 4 $C_{n-}^{1}+4 C_{n-}^{2}+4 C_{a-}^{1}+4 C_{a-}^{2}=336$.

\subsection{Search space reduction}

In this example, the node match (\{restart $\},$ restart $\}$ ) is frozen because (i) it is a perfect match, and (ii) its neighborhood is perfectly matched (\{Lottery $\}$, $\{$ Lottery\}). These nodes and arcs adjacent to them are deemed irrelevant for the matching process.

For each pair of nodes (of the same type), we compute contextual similarity values as illustrated by Table 17 for the pair (\{BuyTciket\}, \{buySomeTickets $\}$ ). Table presents the results for the pairs with a contextual similarity above the fixed threshold (0.5).

\subsection{Similarity computation}

For each of the valid pairs, the function nodeDiff has to be computed. Let us consider the pair $\left(x_{1}, x_{2}\right)=$ (\{BuyTciket $\},\{$ buySomeTickets $\}$ ).

$$
\begin{aligned}
& x_{1} \text { : public Ticket BuyTciket(Lottery) } \\
& \hookrightarrow \text { name }_{1}=\text { BuyTciket }, \\
& \hookrightarrow \text { features }_{1}=[\text { public, } \text { Ticket, } \text { Lottery }] \\
& x_{2} \text { : public MyTicket buySomeTickets (Lottery) } \\
& \hookrightarrow \text { name }_{2}=\text { buySomeTickets, } \\
& \hookrightarrow \text { features }_{2}=[\text { public, MyTicket, Lottery }]
\end{aligned}
$$

\section{Table 18}

Valid pairs after search space reduction $\left(C_{1}, C_{2}\right.$, and $C_{12}$ respectively stand for $T C_{1}, T C_{2}$, and $T C_{1} \cap T C_{2}$ )

\begin{tabular}{l|l|l|l|l|l|l|l}
\hline Node $_{1}$ & Node $_{2}$ & $\left|C_{1}\right|$ & $\left|C_{2}\right|$ & $\left|C_{12}\right|$ & $c S_{1}$ & $c S_{2}$ & nDiff \\
\hline root & root & 6 & 7 & 4 & $\mathbf{0 . 6 7}$ & $\mathbf{0 . 5 7}$ & 0 \\
TheClient & Client & 15 & 11 & 10 & $\mathbf{0 . 6 7}$ & $\mathbf{0 . 9 1}$ & 0.1 \\
TheClient & Ticket & 16 & 3 & 2 & 0.13 & $\mathbf{0 . 6 7}$ & 0.44 \\
TheClient & Distribution & 16 & 3 & 2 & 0.13 & $\mathbf{0 . 6 7}$ & 0.45 \\
Ticket & MyTicket & 11 & 10 & 5 & 0.45 & $\mathbf{0 . 5}$ & 0.07 \\
Ticket & Ticket & 11 & 3 & 3 & 0.27 & $\mathbf{1}$ & 0 \\
Ticket & Distribution & 11 & 4 & 3 & 0.27 & $\mathbf{0 . 7 5}$ & 0.37 \\
Lottery & Lottery & 12 & 16 & 7 & $\mathbf{0 . 5 8}$ & 0.44 & 0 \\
Instance & Client & 3 & 13 & 2 & $\mathbf{0 . 6 7}$ & 0.15 & 0.43 \\
newLottery & addnewLottery & 4 & 4 & 2 & $\mathbf{0 . 5}$ & $\mathbf{0 . 5}$ & 0.07 \\
YouWon & youWon & 6 & 6 & 4 & $\mathbf{0 . 6 7}$ & $\mathbf{0 . 6 7}$ & 0 \\
cTokens & cTickets & 6 & 6 & 3 & $\mathbf{0 . 5}$ & $\mathbf{0 . 5}$ & 0.25 \\
BuyTciket & buySomeTickets & 10 & 11 & 7 & $\mathbf{0 . 7}$ & $\mathbf{0 . 6 3}$ & 0.31 \\
\hline
\end{tabular}

Names generate the following sets of terms: name $_{1} \rightarrow$ \{buy, tciket $\}$ and name $_{2} \rightarrow$ \{buy, some, tickets\}.

Features generate sets of terms grouped according to the feature they represent. For example, for the methods of a class, the features are the modifier, the return type, and the input types. In our example, we have features $_{1} \rightarrow[$ \{public\}, \{ticket $\},\{$ lottery\}] and features $_{2} \rightarrow[$ \{public $\},\{$ my, ticket $\},\{$ lottery $\left.\}\right]$.

Computation of label similarity goes as follows:

textSim $1\left(\right.$ name $_{1}$, name $\left._{2}\right)=\frac{2 \times 1}{2+3}=0.4$

textSim $_{2}\left(\right.$ name $_{1}$, name $\left._{2}\right)=\frac{2 \times 6}{9+14}=0.52($ Table 4$)$

nameSim $\left(\right.$ name $_{1}$, name $\left._{2}\right)=\max (0.4,0.52)=0.52$

textSim $_{1}\left(\right.$ features $_{1}$, feature $\left._{2}\right)=\frac{2 \times 3}{3+4}=0.86$

featSim $\left(\right.$ features $_{1}$, features $\left._{2}\right)=0.86$

For $n w=0.5, f w=0.5$, we obtain

$\operatorname{nodeSim}\left(x_{1}, x_{2}\right)=0.5 \times 0.52+0.5 \times 0.86=0.69$

nodeDiff $\left(x_{1}, x_{2}\right)=1-0.69=0.31$

\subsection{Local Search}

All pairs present in Table 18 are considered for addition or removal from the solution: if the pair is already in the solution, it is removed, otherwise it is added.

1) $+(\{$ TheClient $\},\{$ Client $\}):-96(f(S)=653)$

2) $+(\{$ YouWon $\},\{$ youWon $\}):-84(f(S)=569)$

3) $+(\{$ BuyTciket $\}$, \{buySomeTickets $\}):-85(f(S)=484)$

4) $+(\{$ cTokens $\}$, \{cTickets $\}):-73(f(S)=411)$

5) $+(\{$ newLottery $\}, \quad\{$ addNewLottery $\}) \quad$ : -57 $(\mathrm{f}(\mathrm{S})=354)$

6) $+(\{$ Ticket $\}, \quad$ MyTicket $\}) \Rightarrow$ Merge $(\{$ Ticket $\}$, $\{$ Ticket, MyTicket\}) : $-35(\mathrm{f}(\mathrm{S})=319)$

7) $+(\{$ TheClient $\},\{$ Distribution $\}):+\mathbf{8 1}(\mathbf{f}(\mathbf{S})=\mathbf{4 0 0})$

Subsequent iterations of the search will see tentative merges, deletions and additions of node matches but, ultimately, the solution reached in iteration 6 cannot be improved. The state of the solution at this iteration is the best configuration and the one returned. For a better understanding of the differential cost brought by each move, let us focus on iterations 2 and 6 .

Before Iteration 2, the nodes YouWon and youWon as well as their adjacent arcs (2 for each of these nodes) were unmatched, bearing a cost of $C_{n-}^{1}+C_{n-}^{2}+2 C_{a-}^{1}+2 C_{a-}^{2}$. Just after Iteration 2, these nodes are now 
Table 17

Contextual similarity between BuyTciket and buySomeTickets

\begin{tabular}{c|l|l|l|l|l|l|l|l|l|l|l|l|l}
\hline & buy & tciket & some & tickets & you & won & c & tokens & ticket & my & lottery & the & client \\
\hline BuyTciket & $1,0,0$ & $1,0,0$ & $0,0,0$ & $0,0,0$ & $0,0,1$ & $0,0,1$ & $0,0,1$ & $0,0,1$ & $0,0,1$ & $0,0,0$ & $0,0,1$ & $0,1,0$ & $0,1,0$ \\
buySomeTickets & $1,0,0$ & $0,0,0$ & $1,0,0$ & $1,0,1$ & $0,0,1$ & $0,0,1$ & $0,0,1$ & $0,0,0$ & $0,0,1$ & $0,0,1$ & $0,0,1$ & $0,0,0$ & $0,1,0$ \\
\hline intersection & 1 & 0 & 0 & 0 & 1 & 1 & 1 & 0 & 1 & 0 & 1 & 0 & 1 \\
\hline
\end{tabular}

matched (zero cost instead of $C_{n-}^{1}+C_{n-}^{2}$ ), arcs $\overrightarrow{\{\text { TheClient }\}\{\text { YouWon }\}}$ and $\overrightarrow{\{C l i e n t\}\{y o u W o n}\}$ are perfectly matched (zero cost instead of $\left.C_{a-}^{1}+C_{a-}^{2}\right)$ but arcs $\overrightarrow{\{\text { YouWon }\{\text { BuyTciket }\}}$ and

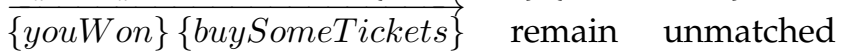
(same cost $C_{a-}^{1}+C_{a-}^{2}$ ). The move thus generated savings of $C_{n-}^{1}+C_{n-}^{2}+C_{a-}^{1}+C_{a-}^{2}=84$.

Before Iteration 6, (\{Ticket $\},\{$ Ticket $\}$ ) is already in the solution, thus adding (\{Ticket\} \{MyTicket $\}$ ) results in a merge in the second graph: (\{Ticket\}, \{Ticket, MyTicket\}). Before the merge, we had ( $\{$ Ticket $\},\{$ Ticket $\}$ ) (cost of 0 ) and MyTicket is unmatched (cost of $C_{n-}^{2}$ ). After the merge, we have to compute nodeDiff( $\{$ Ticket $\},\{$ Ticket, MyTicket $\})$. The name similarity between these nodes is computed using the sets \{ticket\} and $\{$ my, ticket $\}$ and has a value of 0.86 . Feature similarity is 1 , thus nodeDiff $=1$ - $(0.86+1) / 2=0.07$. This value has to be multiplied by $C_{n+}$ and 2 (the size of the super-node); the final value is 14. Similarly, all the arcs linked to these matched nodes may generate differential costs. For instance, the arc $\overrightarrow{\{\text { root }\}\{M y \text { Ticket }\}}$ goes from being previously unmatched (cost is $C_{a-}^{1}=7$ ) to generating a match error $(\overrightarrow{\{\text { root }\}\{\text { Ticket, MyTicket }\}}-\overrightarrow{\{\text { root }\}\{\text { Ticket }\}}=\{9\})$ involving a super-node of size 2 (cost is $\left.2^{*} C_{a-}^{2}=20\right)$; it yields $a+13$ cost differential.

\section{References}

[1] G. Antoniol, G. Canfora, G. Casazza, and A. De Lucia. Maintaining traceability links during object-oriented software evolution. Software - Practice and Experience, 31:331-355, April 2001.

[2] G. Antoniol, M. Di Penta, and E. Merlo. An automatic approach to identify class evolution discontinuities. In IWPSE, pages 31-40, 2004.

[3] D. Binkley, M. Davis, D. Lawrie, and C. Morrell. To camelcase or under_score. In ICPC, pages 158-167, 2009.

[4] K. Bogdanov and N. Walkinshaw. Computing the structural difference between state-based models. In WCRE, pages 177186, 2009.

[5] H. Bunke. On a relation between graph edit distance and maximum common subgraph. Pattern Recognition Letters, 18(9):689-694, 1997.

[6] H. Bunke. Error-tolerant graph matching: a formal framework and algorithms. In Proc. Advances in Pattern Recognition, pages $1-14,1998$.

[7] T. H. Cormen, C. E. Leiserson, and R. L. Rivest. Introductions to Algorithms. MIT Press, 1990.

[8] D. Kimelman D. Mandelin and D.M. Yellin. A bayesian approach to diagram matching with application to architectural models. In ICSE, pages 222-231, 2006.

[9] B. Dagenais and M. P. Robillard. Recommending adaptive changes for framework evolution. In ICSE, pages 481-490, 2008.
[10] M. Torchiano G. Reggio F. Ricca, G. Scanniello and E. Astesiano. On the effectiveness of screen mockups in requirements engineering: results from an internal replication. In ESEM, 2010.

[11] F. Glover. Tabu search-part I. ORSA Journal on Computing, 1 (3):190 - 206, 1989.

[12] M.W. Godfrey and L. Zou. Using origin analysis to detect merging and splitting of source code entities. IEEE Transactions on Software Engineering, 31(2):161-181, Feb. 2005.

[13] Y.-G. Gueheneuc and G. Antoniol. Demima: A multilayered approach for design pattern identification. IEEE Transactions on Software Engineering, 34(5):667-684, 2008.

[14] M. Kim, D. Notkin, and D. Grossman. Automatic inference of structural changes for matching across program versions. In ICSE, pages 333-343, 2007.

[15] D. Kimelman, M. Kimelman, D. Mandelin, and D. M. Yellin. Bayesian approaches to matching architectural diagrams. IEEE Transactions on Software Engineering, 36(2):248-274, 2010.

[16] S. Kpodjedo. Approximate Graph Matching For Software Engineering. Ph.D. Thesis, Ecole Polytechnique de Montreal, Montreal Canada, 092011.

[17] S. Kpodjedo, P. Galinier, and G. Antoniol. Using local similarity measures to efficiently address approximate graph matching. Discrete Applied Mathematics, http://10.1016/j.dam.2012.01.019, 2012.

[18] S. Kpodjedo, F. Ricca, P. Galinier, G. Antoniol, and Y.-G. Gueheneuc. Studying software evolution of large object-oriented software systems using an etgm algorithm. Journal of Software Maintenance and Evolution, http://dx.doi.org/10.1002/smr.519, 2010.

[19] H.W. Kuhn. The hungarian method for the assignment problem. Naval Research Logistics Quarterly, 2(1-2):83-97, 1955.

[20] A. De Lucia, M. Di Penta, and R. Oliveto. Improving source code lexicon via traceability and information retrieval. IEEE Transactions on Software Engineering, 37(2):205-227, 2011.

[21] W.-J. Park and D.-H. Bae. A two-stage framework for uml specification matching. Information $\mathcal{E}$ Software Technology, 53(3):230-244, 2011.

[22] W. N. Robinson and H. G. Woo. Finding reusable uml sequence diagrams automatically. IEEE Software, 21(5):60-67, 2004.

[23] M. Chechik S. Easterbrook S. Nejati, M. Sabetzadeh and P. Zave. Matching and merging of statecharts specifications. In ICSE, pages 54-64, 2007.

[24] A. Sanfeliu and K. S. Fu. A distance measure between attributed relational graphs for pattern recognition. IEEE Transactions on Systems, Man, and Cybernetics, 13:353-362, 1983.

[25] T. Schafer, J. Jonas, and M. Mezini. Mining framework usage changes from instantiation code. In ICSE, pages 471-480, 2008.

[26] W. Tsai and K. S. Fu. Error-correcting isomorphism of attributed relational graphs for pattern analysis. IEEE Trans. on Systems, Man, and Cybernetics, 9:757 - 768, 1979.

[27] Q. Tu and M.W. Godfrey. An integrated approach for studying architectural evolution. In IWPC, pages 127-136. IEEE Computer Society, 2002.

[28] G. Antoniol W. Wu, Y.-G. Gueheneuc and M. Kim. Aura: a hybrid approach to identify framework evolution. In ICSE (1), pages 325-334, 2010.

[29] Z. Xing. Model comparison with genericdiff. In ASE, pages 135-138, 2010.

[30] Z. Xing and E. Stroulia. Analyzing the evolutionary history of the logical design of object-oriented software. IEEE Transactions on Software Engineering, 31(10):850-868, October 2005.

[31] Z. Xing and E. Stroulia. UMLDiff: an algorithm for objectoriented design differencing. In ASE, pages 54-65. ACM, 2005.

[32] Z. Xing and E. Stroulia. Api-evolution support with diffcatchup. IEEE Transactions on Software Engineering, 33(12):818836, Dec. 2007. 